\title{
An Experimental Investigation of Colonel Blotto Games*
}

\author{
Subhasish M. Chowdhury ${ }^{\mathrm{a}}$, Dan Kovenock ${ }^{\mathrm{b}}$, and Roman M. Sheremeta ${ }^{\mathrm{c}}$ \\ ${ }^{a}$ School of Economics, Centre for Behavioral and Experimental Social Science, and ESRC \\ Centre for Competition Policy, University of East Anglia, Norwich NR4 7TJ, UK \\ ${ }^{b}$ Department of Economics, The University of Iowa \\ W284 John Pappajohn Bus Bldg, Iowa City, IA 52242-1994, U.S.A. \\ ${ }^{c}$ Argyros School of Business and Economics, Chapman University, \\ One University Drive, Orange, CA 92866, U.S.A.
}

September 16, 2011

\begin{abstract}
This article examines behavior in the two-player, constant-sum Colonel Blotto game with asymmetric resources in which players maximize the expected number of battlefields won. The experimental results support the main qualitative predictions of the theory. In the auction treatment, where winning a battlefield is deterministic, disadvantaged players use a "guerilla warfare" strategy which stochastically allocates zero resources to a subset of battlefields. Advantaged players employ a "stochastic complete coverage" strategy, allocating random, but positive, resource levels across the battlefields. In the lottery treatment, where winning a battlefield is probabilistic, both players divide their resources equally across all battlefields. However, we also find interesting behavioral deviations from the theory and discuss their implications.
\end{abstract}

JEL Classifications: C72, C91, D72, D74

Keywords: Colonel Blotto, conflict resolution, contest theory, multi-dimensional resource allocation, rent-seeking, experiments.

Corresponding author: Dan Kovenock, dan-kovenock@uiowa.edu

* We have benefitted from the helpful comments of two anonymous referees and an associate editor, Jason Abrevaya, Tim Cason, Ron Harstad, Brian Roberson, seminar participants at the Free University of Berlin, Louisiana State University, Purdue University, and the University of East Anglia, and participants at the 2008 Annual Conference at the Centre for Studies in Social Sciences (Calcutta, India), the 2008 North American Annual ESA Conference, the 2009 Midwest Economic Theory Meetings, and the 2010 Public Economic Theory Conference. This research has been supported by National Science Foundation Grant (SES-0751081). Any remaining errors are ours. 


\section{Introduction}

This article experimentally investigates the classic Colonel Blotto game with asymmetric resources. In this constant-sum game, two players simultaneously allocate their endowments of resources across $n$-battlefields, with the objective of maximizing the expected number of battlefields won. The probability of winning a battlefield depends on the resources allocated by both players to that field. The function that maps the two players' resource allocations into their respective probabilities of winning is called the contest success function (CSF). We examine two types of contest success functions (CSFs): the "auction" CSF, in which the player allocating more resources to a battlefield wins that battlefield with certainty, and the "lottery" CSF, in which the probability of winning a battlefield equals the ratio of a player's resource allocation to the sum of the players' resource allocations in that battlefield.

The experimental results support the main qualitative predictions of the theory. In the auction treatment, where the winner of each battlefield is determined according to the auction CSF, disadvantaged players often use a "guerilla warfare" strategy which stochastically allocates zero resources to a subset of battlefields. Advantaged players often employ a "stochastic complete coverage" strategy, allocating random, but positive, resource levels to each battlefield. Under the lottery treatment, where the winner of each battlefield is determined according to the lottery CSF, there is support for the equilibrium prediction of a constant allocation across battlefields for both players. However, we also find interesting behavioral deviations from the theory. Due to the constant-sum nature of the game, we examine behavior under both strangers and partners matching protocols. In the auction treatment, under the strangers protocol, players have significant serial correlation in allocations to a given battlefield across time. Under the partners protocol this correlation is significantly reduced, and disappears for the disadvantaged 
player. Intuitively, under the auction treatment and partners protocol, both players want to be less predictable (i.e., reduce serial correlation), and this incentive is even stronger for the disadvantaged player (i.e., player whose loss from being predictable is the highest).

The Colonel Blotto game is the prototype of models of multidimensional strategic resource allocation. Originally formulated by Borel (1921), it is among the first strategic situations to be subject to formal mathematical analysis. Over the years, variants of the game have been examined by prominent scholars across a wide range of disciplines (Tukey, 1949; Blackett, 1954, 1958; Bellman, 1969; Shubik and Weber, 1981; Snyder, 1989; Powell, 2007; and Hart, 2008). Interest in the game is derived from its wide potential for application, including to problems in military and systems defense (Blackett, 1954, 1958; Shubik and Weber, 1981; Clark and Konrad 2007; Powell 2007, Hausken, 2008; and Kovenock and Roberson, 2009b), advertising (Friedman, 1958), research and development portfolio selection (Clark and Konrad, 2008), political campaign resource allocation (Snyder, 1989; Klumpp and Polborn, 2006; and Strömberg, 2008) and redistributive politics (Laslier, 2002; Laslier and Picard, 2002; and Roberson, 2008). ${ }^{1}$ The two most popular contest success functions employed in these analyses are the auction and lottery CSFs.

Borel's original version of the Colonel Blotto game employed an auction CSF and was solved for the special case of three battlefields and symmetric resources by Borel and Ville (1938). Gross and Wagner (1950), extended the Borel and Ville analysis of the case of symmetric resources to allow for any finite number of battlefields. Friedman (1958) provided a partial characterization of the solution to Borel's problem for $n$-battlefields and asymmetric resources. More recently, Roberson (2006) has applied the theory of copulas to prove the

\footnotetext{
${ }^{1}$ Conceptually related, but somewhat different technically, are the models of redistributive politics with a continuum of battlefields following Myerson (1993). Contributions in this line of research include Lizzeri (1999), Lizzeri and Persico (2001), Sahuguet and Persico (2006), Crutzen and Sahuguet (2009), and Kovenock and Roberson (2009a).
} 
uniqueness of equilibrium payoffs under the auction CSF for $n$-battlefields and arbitrary asymmetric resources and prove that uniform univariate marginal distributions are necessary for equilibrium over a wide range of endowments of resources. ${ }^{2}$ To our knowledge, Friedman (1958) was also the first to examine the Blotto game under the lottery CSF and solved the game for $n$ battlefields and asymmetric resources. A recent extension is Robson (2005), who extends the analysis from the lottery CSF to more general CSF's of the ratio form in which the probability that player $i$ wins the contest as a function of the two levels of expenditure $x_{1}$ and $x_{2}$ is $x_{i}^{r} /\left(x_{1}^{r}+\right.$ $\left.x_{2}^{r}\right)$, where $0<r \leq 1 .^{3}$

Which of the contest success functions better describes a given strategic multidimensional resource allocation problem depends on the nature of the conflict within each contested battlefield. An auction CSF might well approximate environments in which exogenous noise plays little role in influencing the outcome of the battle. The lottery CSF is a popular method of modeling environments in which victory in a given battlefield is determined not just by the respective resource allocations, but also a substantial random component.

There exists a substantial experimental literature on lottery contests (Davis and Reilly, 1998; Potters et al., 1998; Sheremeta, 2010, 2011; Sheremeta and Zhang, 2010; Morgan et al., forthcoming), as well as all-pay auctions with complete information (Davis and Reilly, 1998; Potters et al., 1998; Gneezy and Smorodinsky, 2006; Lugovskyy et al., 2010). The vast majority

\footnotetext{
${ }^{2}$ See also Kvasov (2007) and Roberson and Kvasov (forthcoming), who examine "non-constant sum" Blotto games in which budgets are not use-it-or-lose-it, Golman and Page (2009) who examine Blotto games with payoffs nonlinear in the number of battlefields won and externalities across battlefields, and Hart (2008) who examines a Blotto game with discrete strategy spaces.

${ }^{3}$ Snyder (1989) examined a related game in which the CSF for each battlefield was of the type employed by Rosen (1986) and contained the lottery CSF as a special case. Snyder assumed no budget constraints, but instead a positive marginal cost of each unit resource employed. He also examined two different objectives, one involving a payoff linear in the number of battlefields won and the other a payoff that was discontinuous when a majority of battlefields was won.
} 
of experimental studies focus on simple single-battle contests. ${ }^{4}$ Almost without exception, the existing studies provide evidence of subjects behaving more aggressively than predicted by the Nash equilibrium. Commonly cited explanations for over-dissipation in contests are that subjects make mistakes (Potters et al., 1998; Sheremeta, 2011; Schmidt et al., 2011), they misperceive probabilities (Amaldoss and Rapoport, 2009; Sheremeta and Zhang, 2010), and they have a nonmonetary utility of winning (Parco et al. 2005; Sheremeta, 2010; Price and Sheremeta, 2011). The unique feature of our study is that our experiments investigate constant-sum contests in which players cannot over-dissipate, whereas all previous studies investigate non-constant-sum contests. In contrast to previous experimental studies on the all-pay auction (i.e., Potters et al., 1998; Gneezy and Smorodinsky, 2006; Lugovskyy et al., 2010), we find that the payoffs in the Colonel Blotto game are very close to the theoretical prediction. But we also find that the pattern of dichotomous behavior (players choosing either very low bids or moderately high bids more often than predicted) is similar to the findings in the all-pay auction literature.

Most closely related to our study is the study by Avrahami and Kareev (2009), who investigate behavior in a discrete Colonel Blotto game with an auction CSF (as in Hart, 2008). Although many of the details of both their experiment and analysis differ from ours, the key difference is that we use a finer mesh of feasible strategies to approximate the continuous Colonel Blotto game (Roberson, 2006). Moreover, we compare two prominent CSFs: lottery versus auction, which represent alternative assumptions about the technology of conflict.

The rest of the article is organized as follows. In section 2, we describe our experimental design and theoretical predictions. Section 3 presents the results of our experiment and compares these results to the corresponding theoretical predictions. Section 4 concludes.

\footnotetext{
${ }^{4}$ Although there are several studies in the literature (see Sheremeta, 2010), investigating multi-battle elimination contests.
} 


\section{Experimental Environment}

\subsection{Experimental Design and Theoretical Predictions}

This article examines experimentally whether behavior conforms to the Nash equilibrium predictions of the Colonel Blotto game with asymmetric budgets. Our experimental design is based on the constant-sum Colonel Blotto game, in which two players simultaneously allocate their resources across $n$-battlefields, with the objective of maximizing the expected number of battlefields won. ${ }^{5}$ We study two treatments: the auction treatment, in which the player with the higher resource allocation to a battlefield wins that battlefield with certainty, and the lottery treatment, in which the probability of winning a battlefield equals the ratio of a player's resource allocation to the sum of the players' resource allocations in that battlefield. The auction treatment is based on Roberson (2006) and the lottery treatment is based on Friedman (1958).

The structure of the game is shown in Figure 1. We use 8 battlefields (boxes) and two players with asymmetric resources. The resource endowment for player 1 is 200 tokens and for player 2 it is 120 tokens. The outline of the experimental design along with the theoretical predictions is shown in Table 1. Under the auction CSF (auction treatment), for the parameters chosen, there exists no pure strategy Nash equilibrium in this game. For 8 battlefields and the ratio of budgets chosen (120/200), Roberson (2006) demonstrates that mixed strategy equilibrium univariate marginal distributions are unique. ${ }^{6}$ We chose 8 battlefields and endowments of 120 and 200 tokens to both ensure uniqueness of these marginal distributions and also to ensure that the allocation problem for the subjects is non-trivial. Endowments were also

\footnotetext{
${ }^{5}$ Since the games examined are constant sum, Nash equilibrium strategies are also optimal strategies.

${ }^{6}$ Roberson (2006) constructs equilibrium $n$-variate distributions for these and all possible parameters configurations, but a complete characterization of the equilibrium set is still an open question.
} 
chosen so that player 2 had a substantial probability of winning some battlefields. Moreover, these parameters, together with private conversion rates, were chosen to ensure equal earnings between player 1 and player 2 .

The qualitative nature of the mixed strategy equilibria that arise depends on the ratio of the two players' budgets. For the budgets examined here, the disadvantaged player 2 allocates zero resources to a given battlefield with positive probability (0.4) and then employs a uniform marginal distribution between zero ( 0 tokens) and a common upper bound (50 tokens). Hence, the disadvantaged player 2 uses a "guerilla warfare" strategy which stochastically allocates zero resources to a subset of battlefields. The advantaged player 1's equilibrium strategy must generate marginal distributions that are uniform over the complete support, which coincides with that of the disadvantaged player. Hence, equilibrium strategies for the advantaged player exhibit "stochastic complete coverage," allocating random, but positive, resource levels across the battlefields. The unique equilibrium expected payoff for the advantaged player is 0.7 and for the disadvantaged player it is 0.3 .

The Colonel Blotto game with the lottery CSF (lottery treatment) applied in each battlefield yields markedly different equilibrium predictions. For all positive budget pairs of the two players, the unique equilibrium requires that players employ pure strategies that divide their budgets equally across the $n$-battlefields (Friedman, 1958). Given the specific parametric restrictions used in our experiment, equilibrium requires that the advantaged player 1 allocates 25 tokens, whereas the disadvantaged player 2 allocates 15 tokens to each box. It is straightforward to calculate the expected payoff in the lottery treatment. The expected payoff per box is equal to the probability of winning that box. Hence, player 1's expected payoff is $25 /(25+15)=0.625$ and player 2 's expected payoff is 0.375 . 


\subsection{Experimental Procedures}

The experiment was conducted at the Vernon Smith Experimental Economics Laboratory during March and May of 2008. The computerized experimental sessions were run using z-Tree (Fischbacher, 2007). A total of 128 subjects participated in eight sessions. All subjects were Purdue University undergraduate students who participated in only one session of this study. Some students had participated in other economics experiments unrelated to this research.

Table 2 summarizes the design of the experiment. We employ two treatment variables: CSF (lottery versus auction) and matching protocol (strangers versus partners). Each experimental session had 16 subjects and it proceeded in two parts, corresponding to the lottery and auction treatments. ${ }^{7}$ Each subject played for 15 periods in the lottery treatment and 15 periods in the auction treatment. The sequence was varied so that half the sessions had the auction treatment first, and half had the lottery treatment first. ${ }^{8}$

At the beginning of each treatment subjects were given instructions, available in the Appendix, and the experimenter read the instructions aloud. Before the first period of the experiment subjects were randomly and anonymously assigned as player 1 or player 2 . All subjects remained in the same role assignment throughout the entire experiment. In sessions 1-4, where we employed the strangers matching protocol, subjects of opposite assignments were

\footnotetext{
${ }^{7}$ Before the start of the experiment we also elicited subjects' risk preferences by utilizing a series of 15 lottery choices as in Holt and Laury (2002). In eliciting risk preferences we used US dollars instead of experimental francs. This was mainly done to be comparable with other studies on contests (Sheremeta 2010, 2011), and we also wanted to know explicitly subjects' risk preferences based on US dollars and not on francs (although theoretically they should be equivalent). However, we did not find any interesting patterns or correlations between risk attitudes and the behavior in the Colonel Blotto game. So, we omit any discussion from the article.

${ }^{8} \mathrm{We}$ did not find any significant order effect, so for the same treatment we combined the data from the first and last 15 periods of the experiment.
} 
randomly re-paired each period to form a two player group. ${ }^{9}$ In sessions $5-8$, where we employed the partners matching protocol, subjects were paired with the same participant of opposite assignment for the entire experiment. In each period, player 1 received 200 tokens and player 2 received 120 tokens. Both players were asked to choose how to allocate their tokens across 8 boxes. Player 1 could allocate any number of tokens between 0 and 200 (with a mesh of 0.5 tokens) to each box. In each period the total number of tokens had to sum to 200 or the computer did not accept the allocation of player 1. A corresponding rule was applied to player 2 up to his budget of 120 tokens. After all subjects in the session submitted their allocations for a given period, the computer informed each player which boxes they had won. The winner of each box received 1 franc (experimental currency). In the lottery treatment, the winner of each box was chosen according to the lottery CSF. A simple lottery was used to explain how the computer chose the winner. In the auction treatment, the player who allocated more tokens to a particular box was chosen as the winner of that box. We also explained to subjects that when both players allocate the same amount to a given box, the computer would always chose player 1 as the winner of that box. ${ }^{10}$ In each period, after all subjects in the session submitted their allocations, the computer displayed on the outcome screen each player's allocation, the allocation of tokens by the player's opponent, the player's period earnings in francs, and the player's cumulative earnings.

At the end of the experiment, subjects were paid for each of the 15 periods in the lottery treatment and each of the 15 periods in the auction treatment. The earnings were converted into

\footnotetext{
${ }^{9}$ Due to the length of the experiment (i.e. 30 periods), we could not employ the perfect strangers matching protocol. Instead, the 16 subjects were randomly re-matched each period and thus, on average, they played a given opponent during approximately 4 periods in the experiment (i.e., 30/8).

${ }^{10}$ This tie-breaking rule is used in Roberson (2006) to avoid having to employ $\varepsilon$-equilibrium arguments. Therefore, to directly test the theory, we decided to implement the same tie-breaking rule as in Roberson (2006). It is important to emphasize, however, that the specification of the tie-breaking rule does not affect the results for the parameters that we chose in our experiment (for a discussion see Roberson, 2006).
} 
US dollars at the end of the experiment. For player 1, the conversion rate was 8 francs to $\$ 1$, and for player 2, the conversion rate was 4 francs to $\$ 1$. Subjects knew that conversion rates were different for players 1 and 2, although the exact conversion rate was private information for each subject. ${ }^{11}$ On average, subjects earned $\$ 25$ each which was paid in cash. The experimental sessions lasted for about 100 minutes.

\section{Results}

\subsection{General Results}

Table 3 summarizes the average allocation of tokens to each box and the average payoff of each player (combined strangers and partners protocols). ${ }^{12}$ The first support for the "Colonel Blotto" theory comes from the fact that the actual payoffs in Table 3 are very close to the predicted payoffs in Table 1. Specifically, the theory predicts that the expected payoffs per box to players 1 and 2, respectively, are 0.63 and 0.37 in the lottery treatment and 0.70 and 0.30 in the auction treatment. The actual respective payoffs are 0.64 and 0.36 in the lottery treatment and 0.71 and 0.29 in the auction treatment. These payoffs are not significantly different from the theoretical predictions. ${ }^{13}$ The findings are consistent with the findings of Avrahami and Kareev (2009), who also document that in the discrete Colonel Blotto game subjects' payoffs are

\footnotetext{
${ }^{11}$ Although conversion rates are different for players 1 and 2, it does not change the equilibrium of the game, since equilibrium strategies are invariant to multiplication of all payoffs by a positive constant. As long as the subjects know that each box is worth the same amount (i.e., 1 franc in our experiment), the equilibrium prediction is not affected by different private conversion rates.

${ }^{12}$ The average allocations to each box and average payoffs are virtually the same under the strangers and partners protocols. For example, the average payoff of player 1 under the strangers versus the partners protocol is 0.65 versus 0.63 in the lottery treatment and 0.70 versus 0.71 in the auction treatment. The corresponding payoffs for player 2 are 0.35 versus 0.37 , and 0.30 versus 0.29 .

${ }^{13}$ Separately for each treatment and type, we estimated a random effects model, with individual subject effects, where the dependent variable is the payoff and the independent variables are a constant and session dummyvariables. A standard Wald test, conducted on the estimates of a random effects model, cannot reject the hypothesis that the constant coefficients are equal to the predicted theoretical values as in Table 1 (all p-values $>0.05$ ).
} 
consistent with the theoretical predictions. This is an important finding in the experimental contest literature because the vast majority of experimental studies document that payoffs in nonconstant-sum contests do not conform to the theoretical predictions (Davis and Reilly, 1998; Gneezy and Smorodinsky, 2006; Sheremeta, 2010, 2011). In contrast to the previous studies, however, our experiments investigate a constant-sum game, where players cannot over-dissipate, and we find that the actual payoffs are consistent with the theoretical payoffs.

We also find that, consistent with the theoretical predictions, player 1's (2's) payoff of $0.71(0.29)$ in the auction treatment is significantly higher (lower) than player 1's (2's) payoff of $0.64(0.36)$ in the lottery treatment. ${ }^{14}$ This is an interesting result because the average allocation of tokens to each box is the same for both treatments (see Table 3). Nevertheless, the more deterministic nature of the auction treatment more heavily favors the stronger player 1, and thus the expected payoff of player 1 in the auction treatment is higher than in the lottery treatment.

In the lottery treatment, equilibrium requires a constant allocation across all boxes for both players (Table 1). To see whether this prediction is supported, Figure 2 displays the distribution of tokens within each box and over all periods in the lottery treatment. There is some support for the equilibrium prediction as the allocations of player 1 are clearly centered at 25 while the allocations of player 2 are centered at 15 . Contrary to the equilibrium predictions, there is substantial variance in the allocation of tokens within each box. ${ }^{15}$ This variance is consistent

\footnotetext{
${ }^{14}$ Separately for each type, we estimate a random effects model, with individual subject effects, where the dependent variable is the payoff and the independent variables are a constant, a treatment dummy-variable, and session dummy-variables. For both types, the treatment dummy-variable is significant ( $\mathrm{p}$-value $<0.01$ ).

${ }^{15}$ Another deviation from the theory is that subjects on average allocate more tokens towards the first boxes 1-4 than towards boxes 5-8 (Table 3). An explanation for this bias may come from framing effects (Druckman, 2001) and the theory of "focal points" (Schelling, 1960). Although all 8 boxes in our experiment were symmetric from a strategic standpoint, they were located in a row from left-to-right. Thus, subjects may identify boxes 1-4 (on the left) as more focal than boxes 5-8 (on the right). One reason for this may be because $90 \%$ of subjects participating in our experiment write from left-to-right (according to a questionnaire). However, we do not find support for this conjecture since there is no significant correlation between the allocation to boxes 1-4 and a language dummy-
} 
with previous experimental findings in the literature on contests with lottery CSFs (Davis and Reilly, 1998; Potters et al., 1998; Sheremeta, 2010, 2011). Bounded rationality is a prominent explanation for the variance in individual decisions; players may commit errors and potentially learn over time. This process is complicated by the probabilistic nature of the lottery CSF. Each period, the computer makes a random draw to determine the winner of each box. The random draw in period $t-1$ may affect a player's behavior in period $t$, which can explain why there is substantial variance in the allocations in all periods of the experiment. ${ }^{16}$ We do find evidence that subjects learn over time. Particularly, we find that the variance of token allocation within each box decreases substantially from the first five to the last five periods of the experiment. ${ }^{17}$ Nevertheless, even in the last five periods of the experiment the variance of allocations is very substantial. Moreover, the proportion of chosen strategies that are equal to the equilibrium strategies ( 25 and 15 for players 1 and 2, respectively) is almost the same in the first five periods as the last five periods of the experiment ( $45 \%$ versus $43 \%$ for player 1 and $34 \%$ versus $37 \%$ for player 2). These findings suggest that it is difficult for subjects to converge to the pure strategy equilibrium in the lottery treatment.

In the auction treatment, equilibrium requires that player 1 employ a joint distribution which generates for each box a uniform marginal distribution over the interval $[0,50]$. On the other hand, player 2's joint distribution generates a marginal distribution in each box that allocates 0 tokens with probability 0.4 , and randomizes uniformly over the interval $[0,50]$ with the remaining probability. From Figure 3 we can see that the aggregate behavior conforms

variable. It is also possible that subjects allocate more to boxes 1-4 simply because at the beginning of each period the input cursor automatically appears in box 1 .

${ }^{16}$ The mechanisms through which period $t-1$ random outcomes influence decisions in period $t$ are described further in section 3.2.

${ }^{17}$ Separately for player 1 and player 2, a standard F-test for the equality of variances rejects the hypothesis that the allocation of tokens has the same variance in the first 5 periods as in the last 5 periods of the experiment ( $p$-value $<$ $0.05)$. 
substantially to the predictions of equilibrium. ${ }^{18}$ The interval over which players randomize is between 0 and 50 , with only $0.5 \%$ of observed allocations above 50 tokens. Consistent with the theory, the advantaged player 1 employs a "stochastic complete coverage" strategy, allocating a random, but positive, number of tokens across the boxes. The disadvantaged player 2 uses a "guerilla warfare" strategy which stochastically allocates zero tokens to a subset of the boxes. Avrahami and Kareev (2009), in independent work, document similar behavior by a disadvantaged player in a discrete Colonel Blotto game. Also, as in studies of non-constant sum contests (Potters et al., 1998; Barut et al, 2002; Gneezy and Smorodinsky, 2006), behavior is more dichotomous than predicted by the equilibrium, with players choosing either very low allocations or moderately high allocations to a given box more often than predicted.

The theoretical predictions as well as the observed behavior in the lottery treatment are very different from the auction treatment. ${ }^{19}$ The difference also comes from the observation that players use decimals in the allocation of tokens in the auction treatment more often than in the lottery treatment. Figure 3 shows the fraction of players who use decimal points in their allocations. In the auction treatment both players 1 and 2 use decimal points significantly more often than in the lottery treatment. ${ }^{20}$ This finding is due to several factors. First, equilibrium under the auction CSF requires nondegenerate marginal distributions with support $[0,50]$.

\footnotetext{
${ }^{18}$ Obviously, the analysis is based on the aggregate level, after combining all subjects' observations in all rounds, and it may not reflect individual behavior. Indeed, substantial evidence exists that individual choices in games with mixed strategies are quite far from equilibrium predictions (Brown and Rosenthal, 1990). This is also the case in our study since, as we document in sections 3.2 and 3.3, subjects exhibit substantial serial correlation of allocations to a given box. Nevertheless, the major purpose of our study is to test general comparative statics predictions of the theory, i.e. the impact of the contest rule on individual behavior.

${ }^{19}$ We conducted a standard F-test, separately for player 1 and player 2, for the equality of token allocation variances (i.e., comparing the variance of distributions in Figures 3.1 and 3.2). The test clearly rejects the hypothesis that allocation of tokens has the same variance in the auction treatment as in the lottery treatment $(\mathrm{p}$-value $<0.05)$. Therefore, it is apparent that subjects behave very differently across these two treatments.

${ }^{20}$ Separately for each type, we estimate a random effects probit model, with individual subject effects, where the dependent variable is whether a subject chose to use a decimal point in a given box or not and the independent variables are a constant, a treatment dummy-variable, and session dummy-variables. For both types, the treatment dummy-variable is significant ( $p$-value $<0.01)$.
} 
Second, the tie-breaking rule under the auction CSF favors player 1, so one might expect player 2 to attempt to avoid ties. Finally, for the parameters we examine, equilibrium in the lottery treatment requires pure strategies that are whole numbers. From Figure 3 we also see that player 2 uses decimal points more often than player 1 . This difference is especially pronounced and significant for the auction treatment. ${ }^{21}$ One explanation of why player 2 in the auction treatment uses decimal points significantly more often than player 1 is that the deterministic nature of the auction treatment and the tie-breaking rule heavily favor the stronger player 1 and require that subjects assigned the role of player 2 use more sophisticated and diverse strategies. Another explanation is that since player 2 is disadvantaged, subjects in this role may have more incentives to experiment with different ways of outbidding their rivals, which could also lead to using decimal points more often.

\subsection{Strangers versus Partners: Serial Correlation and "Hot Box"}

In non-constant sum games repetition with the same set of players (a partners protocol) may change the nature of equilibrium since subjects have incentive to collude (Kreps et al., 1982). A common way to deal with this is to randomly re-group players (a strangers protocol) after each iteration of the game. However, there is no general agreement on how matching protocol influences individual behavior. In public good games, some studies find more cooperation among strangers, some find more by partners, and some fail to find any difference at all (Andreoni and Croson, 2008; Botelho et al., 2009). In auctions, there is some evidence that subjects collude more under the partners matching protocol (Lugovskyy et al., 2010).

\footnotetext{
${ }^{21}$ Separately for each treatment, we estimate a random effects probit model, with individual subject effects, where the dependent variable is whether a subject chose to use a decimal point in a given box or not and the independent variables are a constant, a type dummy-variable, and session dummy-variables. For the action treatment the type dummy-variable is significant ( $p$-value $<0.01$ ), while for the lottery treatment it is not ( $p$-value $=0.23$ ).
} 
In contrast to the standard auction literature (Klemperer, 2002), collusion is not an issue in our experiment since the Colonel Blotto game presented here is a constant-sum game. Every gain for one player is a loss for the other. However, after we ran the first set of experiments using the conventional strangers protocol, we realized that several very interesting behavioral patterns were caused by this matching protocol. Specifically, we found that players have significant serial correlation in allocations to a given box across periods. Note that, although similar findings have been documented previously in two-player zero-sum games (Brown and Rosenthal, 1990; Walker and Wooders, 2001; Camerer, 2003; Crawford and Iriberri, 2007), our study is novel in that players have a very large pure strategy space (the set of non-negative budget balanced allocations to the eight boxes). The very large number of degrees of freedom embodied in this decision problem enables us to examine the serial correlation of allocations within each box.

Serial correlation of allocations to a given box under the strangers matching protocol is clearly illustrated in Figure 4, where we display the allocations to each box of one of the subjects taking the role of player 1 over the 15 periods in the auction treatment. This particular player 1 received the highest payoff among all players under the strangers matching protocol. The size of a bubble in the figure indicates the size of the allocation. For example, the biggest bubbles in the figure correspond to the allocation of 40 tokens, while the smallest bubbles correspond to the allocation of 10 tokens. The "+" or "-" correspond to winning or losing. Note that this player 1 has a tendency to allocate the same amount of tokens to a given box across periods. We have highlighted those allocations that do not change from period $t$-1 to period $t$ (i.e., perfectly correlated). It is worth examining whether this behavior results from faulty randomization across periods or from some type of individual bias. 
To control for individual and period effects, we provide a multivariate analysis. To capture heterogeneity across individuals we use random effects models with individual subject effects. We also include dummy-variables to capture session effects. The regressions are of the following form:

$$
\begin{aligned}
\text { allocation }_{i t n}= & \beta_{0}+\beta_{1} \text { own-lag }_{i(t-1) n}+\beta_{2} \text { own-lag } \\
& +\beta_{4} \times(1-1) n \times \text { win-lag }_{i(t-1) n}+\beta_{3} \text { opponent-lag }_{i(t-1) n}+ \\
& \sum_{h} \beta_{5 h} S_{h}+u_{i}+\varepsilon_{i t},
\end{aligned}
$$

where allocation is player $i$ 's allocation of tokens to the $n$-th box in a period $t$, own-lag denotes player $i$ 's allocation to the same box in the previous period, win-lag denotes whether player $i$ won that box in the previous period, and opponent-lag denotes the opponent's allocation to that box in the previous period. All regressions also include dummy-variables to capture session effects. The results of the estimation under the strangers protocol are presented in Table $4 .^{22}$ In specifications (1) and (2) we use the data from the lottery treatment while in specifications (3) and (4) we use the data from the auction treatment.

In all specifications the own-lag coefficient is positive and significant, indicating the presence of serial correlation. One explanation of why subjects use strategies with serial correlation is that for opponents such correlation may be hard to detect due to the random matching of subjects. It is also possible some subjects believe that all other subjects are of a similar type. Such beliefs may also lead to serial correlation by inducing subjects to employ previously successful strategies in later periods.

To capture the dynamic aspect of the game, we also used an interaction between own-lag and win-lag. The win-lag variable takes on the value of 1 if the subject won the box in period $t-1$ and 0 otherwise. In specifications (3) and (4) the interaction between own-lag and win-lag is

\footnotetext{
${ }^{22}$ We have also re-estimated Table 4 by including a dummy variable for boxes 1-4. The dummy-variable is positive and significant for player 1 but not for player 2, suggesting that player 1 allocates more tokens to boxes 1-4 than to boxes 5-8. All other estimation results are virtually the same and are available from the authors upon request.
} 
positive and significant. We call this the "hot box" effect. ${ }^{23}$ This is a robust finding since in both specifications we control for the number of tokens allocated to each box by the opponent in period $t-1$. The opponent-lag variable is positive and significant only for player 1 , indicating that player 1 allocates more tokens to the boxes where his opponent allocated more tokens in period $t$-1. One explanation of why there is a "hot box" effect only in specifications (3) and (4) might be due to the deterministic nature of the auction treatment. In the auction treatment, winning a specific box is always a result of a higher allocation, and thus subjects may attribute a winning history specifically to the choice of allocation. On the other hand, in the lottery treatment, a higher allocation to a specific box does not necessarily guarantee winning that box, nor does a lower allocation guarantee defeat. As a consequence, subjects may be more reluctant to attribute a winning history to the specific choice of allocation, making them less likely to repeat the allocation.

The presence of serial correlation and a "hot box" effect motivated us to run sessions with the partners matching protocol. Next we examine the behavior of the subjects under the partners protocol. Figure 5 displays the allocations to each box under the partners protocol of one of the subjects taking the role of player 1 over 15 periods in the auction treatment. This particular player 1 received the highest payoff among all players under the partners matching protocol. The biggest bubbles in the figure correspond to the allocation of 50 tokens, while no bubble corresponds to the allocation of 0 tokens. Again, we have highlighted those allocations that do not change from period $t-1$ to period $t$. Note that the striking difference between Figure 4 and Figure 5 is that the number of perfectly correlated allocations is reduced from 77 to 24 . The player whose allocations are displayed in Figure 5 frequently changes the allocation of tokens to

\footnotetext{
${ }^{23}$ This finding resembles a phenomenon known in the gambling literature as a "hot hand" - a belief in a positive autocorrelation of a non-autocorrelated random sequence. For a review see Chau and Phillips (1995) and Croson and Sundali (2005).
} 
a particular box across periods. One explanation of why subjects use strategies with lower levels of serial correlation when playing against the same opponent in every period is that such correlation is more easily observed and exploited by an opponent in the partners protocol. Under the strangers protocol serial correlation may be harder for opponents to detect due to the random matching of subjects.

For a robust comparison, we re-estimated the regressions from Table 4 using all subjects who made their decisions under the partners matching protocol. The results of the estimation, shown in Table 5, support our initial observation. The own-lag coefficient in all specifications is much lower in Table 5 than in Table 4, indicating that serial correlation is reduced under the partners protocol. Moreover, in the auction treatment, the own-lag coefficient is no longer significant for player 2. Intuitively, in the auction treatment under the partners protocol, both players want to be more unpredictable (i.e., reduce serial correlation), and this incentive is even stronger for the disadvantaged player (i.e., player whose loss from being predictable is the highest). Finally, the estimation results in Table 5 indicate that, under the partners protocol, the "hot box" effect disappears for both players.

\title{
3.3. The Determinants of Payoffs: "Good Ol' Rock"
}

\author{
"Lisa: Look, there's only one way to settle this. Rock-paper-scissors. \\ Lisa's brain: Poor predictable Bart. Always takes 'rock'. \\ Bart's brain: Good ol' 'rock'. Nuthin' beats that! \\ Bart: Rock! \\ Lisa: Paper. \\ Bart: D'oh!"
}

-The Simpsons (http://www.snpp.com/episodes/9F16.html)

In a repeated constant-sum game with equilibrium in nondegenerate mixed strategies, employing the same pure strategy in each period is not a good idea because one's opponent will eventually uncover this pattern and employ a best response. This is what happens to Bart in the 
episode of The Simpsons cited above. In the game of Rock-Paper-Scissors with Lisa, he always plays "Rock", which Lisa recognizes from repeated play. As a result Lisa employs the best response "Paper" and always wins the game. Had Bart played Rock-Paper-Scissors against a different person each period, with his past history unobserved by his opponents, then playing his "good ol' rock" strategy would be difficult to exploit and one would not expect Bart to perform poorly.

In the context of our analysis, a subject playing a pure strategy or some other strategy with high serial correlation in the repeated game would be expected to perform more poorly under the partners protocol than under the strangers protocol. Under the strangers protocol, however, independent re-matching of subjects in each period and the fact that the identity of a subject's current opponent cannot be attached to specific past actions before current play, make it difficult for opponents to detect patterns of play that would be quickly exploited under the partners protocol.

Our use of "good 'ol rock" in the context of the Colonel Blotto game refers to a constant allocation to a given box over time and not to a constant octuple of allocations over time. Given this definition, we found that the "good ol' rock" strategy was frequently employed by subjects in the auction treatment under the strangers protocol. As a general rule, when subjects maintained a constant within-box allocation across periods for one or more of the eight boxes, they did so over a strict subset of the boxes. This type of behavior was significantly reduced when we employed the partners protocol. Support for this finding can be found in Figure 6 which displays the cumulative distribution of the absolute differences between allocations within the same box in periods $t$ and $t+1$ in the auction treatment. Note that the empirical CDF of these within-box absolute differences under the partners protocol first order dominates the 
corresponding CDF under the strangers protocol. When paired with strangers, in $52 \%$ of the time periods subjects in the role of player 1 do not change their allocation from $t$ to $t+1$ (the difference within the same box is 0). However, when paired up with the same partner over time, the percentage goes down to around $18 \%$. For subjects in the role of player 2 , the corresponding percentage changes from $36 \%$ to $24 \%$. These differences are significant based on the estimation of a random effects probit model. ${ }^{24}$ This preliminary analysis provides initial support for our earlier conjecture that the "good ol' rock" strategy is more frequently employed by subjects under the strangers matching protocol than under the partners protocol.

Another interesting result under the two different protocols is the effect of randomization on payoffs. We find that deviations from equilibrium behavior by employing either greater dispersion of resources across boxes in the lottery treatment or less dispersion across boxes (or within each box, across time) in the auction treatment are associated with lower payoffs. To show this, we estimate random effects models of the following form:

$$
\begin{aligned}
\text { payoff }_{i t}=\beta_{0} & +\beta_{1} \text { between-boxes }_{i t}+\beta_{2} \text { between }^{\wedge} 2 \text {-boxes }_{i t}+\beta_{3} \text { within-boxes }_{i t}+ \\
& +\beta_{4 \text { within }^{\wedge} 2-\text { boxes }_{i t}}+\beta_{5}(1 / t)+\sum_{h} \beta_{6 h} S_{h}+u_{i}+\varepsilon_{i t}
\end{aligned}
$$

where payoff is player $i$ 's payoff in a period $t$. The between-boxes variable is defined as the absolute difference between the tokens allocated to a specific box and the mean across all boxes. So, for player 1 (player 2) this variable indicates how far the allocation to a specific box is from 25 (15). The between ${ }^{\wedge} 2$-boxes variable is defined as the square of the difference between the tokens allocated to a specific box and the mean. The within-boxes variable is defined as the absolute difference between the tokens allocated to the same box in periods $t$ and $t-1$. The

\footnotetext{
${ }^{24}$ Separately for each type, we estimate a random effects probit model, with individual subject effects, where the dependent variable is whether the difference within the same box from $t$ to $t+1$ is 0 and the independent variables are a constant, a matching protocol dummy-variable, and session dummy-variables. For both types, the matching protocol dummy-variable is significant ( $\mathrm{p}$-value $<0.05$ ).
} 
within 2 -boxes variable is defined as the square of the difference between the tokens allocated to the same box in period $t$ and period $t-1$.

The estimation results for treatments which used the strangers protocol are shown in Table 6. The between-boxes coefficient in specifications (1) and (2) is negative and significant, indicating that in the lottery treatment there is a strong incentive for players 1 and 2 to converge to the equilibrium allocations of 25 and 15 , respectively. ${ }^{25}$ On the other hand, the between-boxes coefficient in the auction specifications (3) and (4) is positive and significant, indicating that in the auction treatment players 1 and 2 earn significantly higher payoffs by deviating from the mean. To control for the fact that too great a deviation from the mean can actually be harmful, in specifications (5) and (6), we include the between ${ }^{\wedge} 2$-boxes and the within^2-boxes variables. The between-boxes coefficient is still positive and significant, while the between^2-boxes coefficient is negative, indicating that some deviation from the mean increases payoff while excessive deviation decreases payoff. The within-boxes and within^2-boxes variables are not significant in any of the specifications, indicating that the "good ol' rock" strategy does not affect the payoff and thus can be optimal under the strangers protocol. ${ }^{26}$

Table 7 reports the estimation results under the partners protocol. An interesting contrast with Table 6 is reflected in the estimation of specifications (3) through (6). Because of the partners protocol, the "good ol' rock" strategy does not work well in the auction treatment. This is reflected in specifications (3) and (4) by the significant within-boxes coefficient. Randomizing allocations within the same box across periods significantly increases the payoff for both players.

\footnotetext{
${ }^{25}$ By definition, a deviation from equilibrium play leads to a lower payoff when the other player employs the equilibrium strategy. However, when both players simultaneously deviate from the equilibrium strategy it is not obvious what the effect is. That is why we re-estimated the regressions in Tables 6 and 7 without separating the regressions by the players' identity. The estimation results, available upon request, are very similar to those reported.

${ }^{26}$ We have also examined whether the time spent by subjects in making decisions is correlated with payoffs. We reestimated Tables 6 and 7, and found that in only 1 out of 12 specifications was time marginally significant, with a negative coefficient. The results of the estimation are available from authors upon request.
} 
Specifications (5) and (6) confirm that large variation in allocations, even within box, has a negative effect on payoff.

\section{Conclusions}

This article experimentally investigates the classic Colonel Blotto game employing two popular contest success functions: the auction and lottery CSFs. Under the lottery treatment, the equilibrium prediction is that each player should divide their resources equally across all battlefields. The experimental results support this prediction. Moreover, deviations from equilibrium behavior result in lower payoffs. Under the auction treatment, equilibrium requires that the disadvantaged player stochastically allocate zero resources to a subset of battlefields and the advantaged player allocate random, but positive, resource levels across the battlefields. Again, the data support this theoretical prediction and deviations from equilibrium behavior in the form of strategies exhibiting low dispersion of allocations across battlefields at a point in time or within a battlefield over time are associated with lower payoffs. Although the qualitative predictions of the theory in terms of aggregate behavior, payoffs and comparative statics are supported in our experiment, we also find significant behavioral deviations from the theory.

Due to the constant-sum nature of the game, we examined both partners and strangers matching protocols. The choice of matching protocol has significant effects on subject behavior under the auction treatment. Under the strangers protocol subjects are prone to "hot box" and "good 'ol rock" strategies. In the former, winning a box in a period encourages the subject to allocate more resources to that box in the next period. In the latter, independent randomization across periods is replaced with strategies exhibiting high within-box serial correlation of allocations. In fact, under the strangers protocol subjects often allocate exactly the same level of 
the resource to a given box across periods. Occurrence of both the "hot box" and "good ol' rock" strategies significantly diminishes under the partners protocol. To our knowledge, this is the first study to explicitly recognize such effects of the strangers protocol in constant-sum games of multidimensional resource allocation. Our results signal the need for further analyses of these issues.

The Colonel Blotto game is one that is easy to understand yet analytically quite challenging. Because of its compelling structure as a prototype model of strategic multidimensional resource allocation, the game has been utilized in many real-life applications, such as military conflicts, advertising resource allocation, political campaigns, and research and development portfolio selection. Although researchers have been grappling with an analytical solution to the game since Borel (1921), and a complete characterization of the set of equilibria under the auction treatment is still an open question, it took only one hour for subjects who were unfamiliar with this game to exhibit behavior consistent with equilibrium. Players' marginal distributions and payoffs conformed to what we know must be true of all equilibria in the Colonel Blotto game (Roberson, 2006).

The success of experimental results supporting existing theory in this computationally challenging game is very encouraging. It also suggests that experiments can be used extensively to provide guidance for other theoretically challenging problems arising in related games. This remains a promising avenue for the future research. 


\section{References}

Amaldoss, W., \& Rapoport, A.: Excessive expenditure in two-stage contests: Theory and experimental evidence. In F. Columbus (Ed.), Game Theory: Strategies, Equilibria, and Theorems. Hauppauge, NY: Nova Science Publishers (2009)

Andreoni, J., \& Croson, R.: Partners versus Strangers: The Effect of Random Rematching in Public Goods Experiments, in Handbook of Experimental Economics Results, Plott \& Smith eds (2008)

Avrahami, J., \& Kareev, Y.: Do the Weak Stand a Chance? Distribution of Resources in a Competitive Environment. Cognitive Science, 33, 940-950 (2009)

Barut, Y., \& Kovenock, D., \& Noussair, C.N.: A Comparison of Multiple-Unit All-Pay and Winner-Pay Auctions Under Incomplete Information. International Economic Review, 43, 675-708 (2002)

Bellman, R.: On Colonel Blotto and Analogous Games. Siam Review, 11, 66-68 (1969)

Blackett, D.W.: Some Blotto games. Naval Research Logistics Quarterly, 1, 55-60 (1954)

Blackett, D.W.: Pure Strategy Solutions to Blotto Games. Naval Research Logistics Quarterly, 5, 107-109 (1958)

Borel, E.: La theorie du jeu les equations integrales a noyau symetrique. Comptes Rendus del Academie 173, 1304-1308 (1921); English translation by Savage, L.: The theory of play and integral equations with skew symmetric kernels. Econometrica 21, 97-100 (1953)

Borel, E., Ville, J.: Application de la theorie des probabilities aux jeux de hasard. Paris: Gauthier-Villars (1938); reprinted in Borel E., Cheron, A.: Theorie mathematique du bridge a la portee de tous. Paris: Editions Jacques Gabay (1991)

Botelho, A., Harrison, G.W., Pinto, L.M.C., \& Rutström, E.E.: Testing static game theory with dynamic experiments: A case study of public goods. Games and Economic Behavior, 67, 253-265 (2009)

Brown, J.N., \& Rosenthal, R.W.: Testing the Minimax Hypothesis: A Re-examination of O'Neill's Game Experiment. Econometrica, 1065-1081 (1990)

Camerer, C.F.: Behavioral Game Theory: Experiments on Strategic Interaction. Princeton: Princeton University Press (2003)

Chau, A., \& Phillips, J.: Effects of Perceived Control Upon Wagering and Attributions in Computer Blackjack. The Journal of General Psychology, 122, 253-269 (1995)

Clark, D.J., \& Konrad, K.A.: Asymmetric Conflict: Weakest Link against Best Shot. Journal of Conflict Resolution, 51, 457-469 (2007)

Clark, D.J., \& Konrad, K.A.: Fragmented Property Rights and Incentives for R\&D. Management Science, 54, 969-981 (2008)

Crawford, V.P., \& Iriberri, N.: Fatal Attraction: Salience, Naïveté, and Sophistication in Experimental "Hide-and-Seek" Games. American Economic Review, 97, 1731-1750 (2007)

Croson, R., \& Sundali, J.: The Gambler's Fallacy and the Hot Hand: Empirical Data from Casinos, Journal of Risk and Uncertainty, 30, 195-209 (2005)

Crutzen, B.S.Y. \& Sahuguet, N.: Redistributive Politics with Distortionary Taxation. Journal of Economic Theory, 144, 264-279 (2009)

Davis, D., \& Reilly, R.: Do Many Cooks Always Spoil the Stew? An Experimental Analysis of Rent Seeking and the Role of a Strategic Buyer. Public Choice, 95, 89-115 (1998)

Druckman, J.: Evaluating framing effects. Journal of Economic Psychology, 22, 96-101 (2001) 
Fischbacher, U.: z-Tree: Zurich Toolbox for Ready-made Economic Experiments. Experimental Economics, 10, 171-178 (2007)

Friedman, L.: Game-theory Models in the Allocation of Advertising Expenditure. Operations Research, 6, 699-709 (1958)

Gneezy, U., \& Smorodinsky, R.: All-Pay Auctions - An Experimental Study. Journal of Economic Behavior and Organization, 61, 255-275 (2006)

Golman, R., \& Page, S.E.: General Blotto: Games of Strategic Allocative Mismatch. Public Choice, 138, 279-299 (2009)

Gross, O., \& Wagner, R.: A Continuous Colonel Blotto game. Unpublished article, RAND Corporation RM-408 (1950)

Hart, S.: Discrete Colonel Blotto and General Lotto Games. International Journal of Game Theory, 36, 441-460 (2008)

Hausken, K.: Strategic Defense and Attack for Series and Parallel Reliability Systems. European Journal of Operational Research, 186, 856-881 (2008)

Holt, C.A., \& Laury, S.K.: Risk Aversion and Incentive Effects. American Economic Review, 92, 1644-55 (2002)

Klemperer, P.: How (Not) to Run Auctions: The European 3G Telecom Auctions. European Economic Review, 46, 829-845 (2002)

Klumpp, T., \& Polborn, M.K.: Primaries and the New Hampshire Effect. Journal of Public Economics, 90, 1073-1114 (2006)

Kovenock, D., \& Roberson, B.: Inefficient Redistribution and Inefficient Redistributive Politics. Public Choice, 139, 263-272 (2009a)

Kovenock, D., \& Roberson, B.: The Optimal Defense of Networks of Targets. Purdue University, Working Paper (2009b)

Kreps, D., Milgrom, P., Roberts, J., \& Wilson, R.: Rational Cooperation in the Finitely Repeated Prisoners' Dilemma. Journal of Economic Theory, 27, 245-52 (1982)

Kvasov, D.: Contests with limited resources. Journal of Economic Theory, 127, 738-748 (2007)

Laslier, J.F.: How Two-Party Competition Treats Minorities. Review of Economic Design, 7, 297-307 (2002)

Laslier, J.F., Picard, N.: Distributive Politics and Electoral Competition. Journal of Economic Theory, 103, 106-130 (2002)

Lizzeri, A.: Budget Deficits and Redistributive Politics. Review of Economic Studies, 66, 909-28 (1999)

Lizzeri, A., \& Persico, N.: The Provision of Public Goods under Alternative Electoral Incentives. American Economic Review, 91, 225-239 (2001)

Lugovskyy, V., Puzzello, D., \& Tucker, S.: An Experimental Investigation of Overdissipation in the All Pay Auction. European Economic Review, 54, 974-997 (2010)

Morgan, J., Orzen, H. \& Sefton, M.: Endogenous Entry in Contests. Economic Theory, (2011, forthcoming)

Myerson, R.B.: Incentives to Cultivate Minorities under Alternative Electoral Systems. American Political Science Review, 87, 856-869 (1993)

Parco, J., Rapoport, A., and Amaldoss, W.: Two-stage contests with budget constraints: An experimental study. Journal of Mathematical Psychology, 49, 320-338 (2005)

Potters, J.C., De Vries, C.G., \& Van Linden, F.: An Experimental Examination of Rational Rent Seeking. European Journal of Political Economy, 14, 783-800 (1998) 
Powell, R.: Defending against Terrorist Attacks with Limited Resources. American Political Science Review, 101, 527-541 (2007)

Price, C.R., \& Sheremeta, R.M.: Endowment Effects in Contests. Economics Letters, 111, $217-$ $219(2011)$

Roberson, B. \& Kvasov, D.: The Non-Constant Sum Colonel Blotto Game. Economic Theory, (2011, forthcoming)

Roberson, B.: The Colonel Blotto game. Economic Theory 29 (1), 1-24 (2006)

Roberson, B.: Pork-Barrel Politics, Targetable Policies, and Fiscal Federalism. Journal of the European Economic Association, 819-844 (2008)

Robson, A. R. W.: Multi-item contests. Australian National University, Working Paper (2005)

Rosen, S.: Prizes and Incentives in Elimination Tournaments. American Economic Review, 76, 701-715 (1986)

Sahuguet, N., \& Persico, N.: Campaign Spending Regulation in a Model of Redistributive Politics. Economic Theory, 28, 95-124 (2006)

Schelling, T. C.: The Strategy of Conflict. Cambridge, Massachusetts: Harvard University Press (1960)

Schmidt, D., Sheremeta, R.M., Shupp, R., \& Walker, J.: Resource Allocation Contests: Experimental Evidence. Indiana University, Working Paper (2011)

Sheremeta, R.M.: Multi-Stage Elimination Contests: An Experimental Investigation. Games and Economic Behavior, 68, 731-747 (2010)

Sheremeta, R.M.: Contest Design: An Experimental Investigation. Economic Inquiry, 49, 573$590(2011)$

Sheremeta, R.M., \& Zhang, J.: Can Groups Solve the Problem of Over-Bidding in Contests? Social Choice and Welfare, 35, 175-197 (2010)

Shubik, M., \& Weber, R.J.: Systems Defense Games: Colonel Blotto, Command and Control. Naval Research Logistics Quarterly, 28, 281-287 (1981)

Snyder, J.M.: Election Goals and the Allocation of Campaign Resources. Econometrica, 57, 637660 (1989)

Strömberg, D.: How the Electoral College Influences Campaigns and Policy: The Probability of Being Florida. American Economic Review, 98, 769-807 (2008)

Tukey, J.W.: A Problem of Strategy. Econometrica, 17, 73 (1949)

Walker, M., \& Wooders, J.: Minimax Play at Wimbledon. American Economic Review, 91, 1521-1538 (2001) 


\section{Tables and Figures}

Table 1: Experimental Design and Theoretical Predictions

\begin{tabular}{cccccc}
\hline \hline Treatment & $\begin{array}{c}\text { Number } \\
\text { of Boxes }\end{array}$ & Player & Budget & $\begin{array}{c}\text { Equilibrium Marginal } \\
\text { Distribution of Tokens }\end{array}$ & $\begin{array}{c}\text { Expected Payoff } \\
\text { per Box }\end{array}$ \\
\hline Lottery & 8 & 1 & 200 & $x_{1}=25$ & 0.625 \\
& 2 & 2 & 120 & $x_{2}=15$ & 0.375 \\
\hline \multirow{2}{*}{ Auction } & 8 & 1 & 200 & $F_{1}(x)=\frac{1}{50} x \quad$ where $x \in[0,50]$ & 0.7 \\
& \multirow{2}{*}{8} & 120 & $F_{2}(x)=\frac{2}{5}+\frac{3}{250} x$ where $x \in[0,50]$ & 0.3 \\
\hline
\end{tabular}

Table 2: Experimental Design

\begin{tabular}{ccccc}
\hline \hline $\begin{array}{c}\text { Session } \\
\text { Number }\end{array}$ & Design & $\begin{array}{c}\text { Matching } \\
\text { Protocol }\end{array}$ & $\begin{array}{c}\text { Participants } \\
\text { per Session }\end{array}$ & $\begin{array}{c}\text { Periods } \\
\text { per Treatment }\end{array}$ \\
\hline $1-2$ & Lottery $\rightarrow$ Auction & Strangers & 16 & 15 \\
$3-4$ & Auction $\rightarrow$ Lottery & Strangers & 16 & 15 \\
$5-6$ & Lottery $\rightarrow$ Auction & Partners & 16 & 15 \\
$7-8$ & Auction $\rightarrow$ Lottery & Partners & 16 & 15 \\
\hline
\end{tabular}

Table 3: Average Allocations and Payoffs (Strangers and Partners)

\begin{tabular}{|c|c|c|c|c|c|c|c|c|}
\hline \multirow{3}{*}{$\begin{array}{c}\text { Field } \\
1\end{array}$} & \multicolumn{4}{|c|}{ Lottery } & \multicolumn{4}{|c|}{ Auction } \\
\hline & \multicolumn{2}{|c|}{ Player 1} & \multicolumn{2}{|c|}{ Player 2} & \multicolumn{2}{|c|}{ Player 1} & \multicolumn{2}{|c|}{ Player 2} \\
\hline & 25.9 & $(7.6)$ & 16.3 & $(10.9)$ & 26.2 & $(11.9)$ & 16.2 & $(15.8)$ \\
\hline 2 & 25.5 & (7.0) & 15.7 & $(9.4)$ & 26.0 & (11.3) & 14.6 & (15.3) \\
\hline 3 & 26.6 & (7.6) & 15.1 & $(9.2)$ & 27.0 & (10.9) & 16.2 & (15.4) \\
\hline 4 & 25.1 & (7.2) & 14.6 & (9.1) & 25.2 & (11.2) & 14.8 & (14.7) \\
\hline 5 & 25.9 & (6.8) & 15.0 & (9.5) & 26.0 & (10.9) & 15.2 & (15.0) \\
\hline 6 & 23.9 & (7.2) & 15.1 & (8.7) & 24.2 & (10.8) & 15.6 & (15.1) \\
\hline 7 & 23.5 & (7.6) & 14.5 & (8.9) & 23.0 & (11.5) & 15.4 & (14.8) \\
\hline 8 & 23.5 & $(9.0)$ & 13.7 & (8.9) & 22.3 & (12.5) & 12.0 & (14.0) \\
\hline $\begin{array}{c}\text { Payoff } \\
\text { per Box }\end{array}$ & 0.64 & $(0.17)$ & 0.36 & $(0.17)$ & 0.71 & $(0.13)$ & 0.29 & $(0.13)$ \\
\hline
\end{tabular}

Table 4: Determinants of Allocation to a Specific Box (Strangers)

\begin{tabular}{lcccc}
\hline \hline Treatments & Lottery & Lottery & Auction & Auction \\
\hline Dependent variable, & $(1)$ & $(2)$ & $(3)$ & $(4)$ \\
allocation of tokens & Player 1 & Player 2 & Player 1 & Player 2 \\
\hline own-lag & $0.36^{* *}$ & $0.25^{* *}$ & $0.31^{* *}$ & $0.16^{* *}$ \\
[own tokens in period $t$-1] & $(0.02)$ & $(0.02)$ & $(0.03)$ & $(0.03)$ \\
own-lag x win-lag & 0.01 & 0.02 & $0.05^{*}$ & $0.07^{* *}$ \\
$\quad$ [own tokens if subject won the field in period $t-1]$ & $(0.01)$ & $(0.02)$ & $(0.02)$ & $(0.03)$ \\
opponent-lag & $0.06^{* *}$ & 0.03 & $0.10^{* *}$ & -0.05 \\
[opponent's tokens in period $t$-1] & $(0.01)$ & $(0.02)$ & $(0.02)$ & $(0.03)$ \\
1/t & -0.03 & -0.03 & 0.04 & 0.14 \\
[inverse of a period trend] & $(1.00)$ & $(1.34)$ & $(1.38)$ & $(2.02)$ \\
Constant & $14.81^{* *}$ & $10.48^{* *}$ & $14.61^{* *}$ & $13.28^{* *}$ \\
& $(0.50)$ & $(0.67)$ & $(0.58)$ & $(0.88)$ \\
\hline Observations & 3584 & 3584 & 3584 & 3584 \\
Number of subjects & 32 & 32 & 32 & 32 \\
\hline * significant at 5\%, $* *$ significant at $1 \%$. All models include a random effects error structure, with \\
individual subject effects. Each regression also includes dummies, controlling for session effects. \\
We use 3584 observations for estimation of each regression (32 subjects of each type, making 8 \\
decisions in each period, for a total of 15 periods minus 1 period taken by the lag variables).
\end{tabular}


Table 5: Determinants of Allocation to a Specific Box (Partners)

\begin{tabular}{lcccc}
\hline \hline Treatments & Lottery & Lottery & Auction & Auction \\
\hline Dependent variable, & $(1)$ & $(2)$ & $(3)$ & $(4)$ \\
allocation of tokens & Player 1 & Player 2 & Player 1 & Player 2 \\
\hline own-lag & $0.08^{* *}$ & $0.17 * *$ & $0.12^{* *}$ & 0.01 \\
$\quad$ own tokens in period $t$-1] & $(0.02)$ & $(0.02)$ & $(0.03)$ & $(0.03)$ \\
own-lag x win-lag & -0.01 & -0.00 & -0.03 & -0.04 \\
$\quad$ own tokens if subject won the field in period $t-1]$ & $(0.01)$ & $(0.02)$ & $(0.03)$ & $(0.03)$ \\
opponent-lag & $0.03 *$ & 0.01 & 0.00 & $-0.05^{*}$ \\
$\quad$ [opponent's tokens in period $t$-1] & $(0.01)$ & $(0.02)$ & $(0.02)$ & $(0.02)$ \\
1/t & 0.04 & -0.00 & -0.11 & 0.00 \\
$\quad$ inverse of a period trend] & $(1.00)$ & $(1.24)$ & $(1.77)$ & $(2.22)$ \\
constant & $22.84 * *$ & $12.27 * *$ & $22.57^{* *}$ & $16.51^{* *}$ \\
& $(0.50)$ & $(0.62)$ & $(0.59)$ & $(0.76)$ \\
\hline Observations & 3584 & 3584 & 3584 & 3584 \\
Number of subjects & 32 & 32 & 32 & 32 \\
\hline * significant at 5\%, $*$ significant at $1 \%$. All models include a random effects error structure, with
\end{tabular}

Table 6: Determinants of Payoff (Strangers)

\begin{tabular}{|c|c|c|c|c|c|c|}
\hline Treatments & Lottery & Lottery & Auction & Auction & Auction & Auction \\
\hline \multirow{2}{*}{$\begin{array}{l}\text { Dependent variable, } \\
\text { payoff }\end{array}$} & (1) & (2) & (3) & (4) & (5) & (6) \\
\hline & Player 1 & Player 2 & Player 1 & Player 2 & Player 1 & Player 2 \\
\hline \multirow{4}{*}{$\begin{array}{l}\text { between-boxes } \\
\text { [difference between } 8 \text { fields in period } t] \\
\text { between }^{\wedge} 2 \text {-boxes } \\
\quad[\text { difference squared between } 8 \text { fields in period } t]\end{array}$} & $-0.46^{*}$ & $-1.00^{* *}$ & $0.78^{* *}$ & $1.40^{* *}$ & $2.31 * *$ & $2.84^{* *}$ \\
\hline & $(0.20)$ & $(0.16)$ & $(0.20)$ & $(0.17)$ & $(0.43)$ & $(0.44)$ \\
\hline & & & & & $-0.68 * *$ & $-0.61 * *$ \\
\hline & & & & & $(0.22)$ & $(0.18)$ \\
\hline \multirow{4}{*}{$\begin{array}{l}\text { within-boxes } \\
\text { [difference within the same field in periods } t \text { and } t-1] \\
\text { within^ } 2 \text {-boxes } \\
\text { [difference squared within the same field in periods } t \text { and } t-1]\end{array}$} & 0.22 & 0.13 & -0.03 & 0.05 & 0.29 & 0.11 \\
\hline & $(0.17)$ & $(0.14)$ & $(0.13)$ & $(0.07)$ & $(0.27)$ & $(0.25)$ \\
\hline & & & & & -0.12 & -0.01 \\
\hline & & & & & $(0.10)$ & $(0.07)$ \\
\hline \multirow{4}{*}{$\begin{array}{l}\text { [inverse of a period trend }] \\
\text { constant }\end{array}$} & -0.33 & 0.38 & $1.04 *$ & 0.29 & $1.37 * *$ & 0.26 \\
\hline & $(0.51)$ & $(0.48)$ & $(0.43)$ & $(0.38)$ & $(0.41)$ & $(0.39)$ \\
\hline & $5.09 * *$ & $3.33 * *$ & $4.92 * *$ & $0.79 * *$ & $4.53 * *$ & 0.22 \\
\hline & $(0.16)$ & $(0.15)$ & $(0.19)$ & $(0.24)$ & $(0.18)$ & $(0.28)$ \\
\hline \multirow{2}{*}{$\begin{array}{l}\text { Observations } \\
\text { Number of subjects }\end{array}$} & 448 & 448 & 448 & 448 & 448 & 448 \\
\hline & 32 & 32 & 32 & 32 & 32 & 32 \\
\hline
\end{tabular}

Table 7: Determinants of Payoff (Partners)

\begin{tabular}{|c|c|c|c|c|c|c|}
\hline Treatments & Lottery & Lottery & Auction & Auction & Auction & Auction \\
\hline \multirow{2}{*}{$\begin{array}{l}\text { Dependent variable, } \\
\text { payoff }\end{array}$} & (1) & (2) & (3) & (4) & (5) & (6) \\
\hline & Player 1 & Player 2 & Player 1 & Player 2 & Player 1 & Player 2 \\
\hline \multirow{4}{*}{$\begin{array}{l}\text { between-boxes } \\
\quad[\text { difference between } 8 \text { fields in period } t] \\
\text { between } 2 \text {-boxes } \\
\quad[\text { difference squared between } 8 \text { fields in period } t]\end{array}$} & $-0.53^{*}$ & $-0.71 * *$ & 0.11 & $0.64 * *$ & $0.87^{*}$ & 0.57 \\
\hline & $(0.25)$ & $(0.19)$ & $(0.15)$ & $(0.17)$ & $(0.40)$ & $(0.35)$ \\
\hline & & & & & -0.27 & 0.15 \\
\hline & & & & & $(0.18)$ & $(0.13)$ \\
\hline \multirow{4}{*}{$\begin{array}{l}\text { within-boxes } \\
\text { [difference within the same field in periods } t \text { and } t-1] \\
\text { within^2-boxes } \\
\text { [difference squared within the same field in periods } t \text { and } t-1]\end{array}$} & $0.39 *$ & 0.02 & $0.31^{*}$ & $0.24 *$ & $1.18 * *$ & $1.08 * *$ \\
\hline & (0.19) & $(0.17)$ & $(0.13)$ & $(0.10)$ & $(0.28)$ & $(0.23)$ \\
\hline & & & & & $-0.24 * *$ & $-0.23 * *$ \\
\hline & & & & & $(0.07)$ & $(0.06)$ \\
\hline \multirow{4}{*}{$\begin{array}{l}\text { [inverse of a period trend }] \\
\text { constant }\end{array}$} & -0.73 & 0.74 & 0.45 & -0.05 & 0.47 & -0.17 \\
\hline & $(0.49)$ & $(0.49)$ & $(0.41)$ & (0.39) & $(0.38)$ & $(0.39)$ \\
\hline & $5.12 * *$ & $3.23 * *$ & $5.24 * *$ & $1.41 * *$ & $4.67 * *$ & $0.99 * *$ \\
\hline & $(0.20)$ & $(0.18)$ & $(0.18)$ & $(0.22)$ & $(0.20)$ & $(0.26)$ \\
\hline \multirow{2}{*}{$\begin{array}{l}\text { Observations } \\
\text { Number of subjects }\end{array}$} & 448 & 448 & 448 & 448 & 448 & 448 \\
\hline & 32 & 32 & 32 & 32 & 32 & 32 \\
\hline
\end{tabular}

* significant at $5 \%,{ }^{* *}$ significant at $1 \%$. All models include a random effects error structure, with individual subject effects. Each regression also includes dummies, controlling for session effects. 
Figure 1: The Structure of the Game

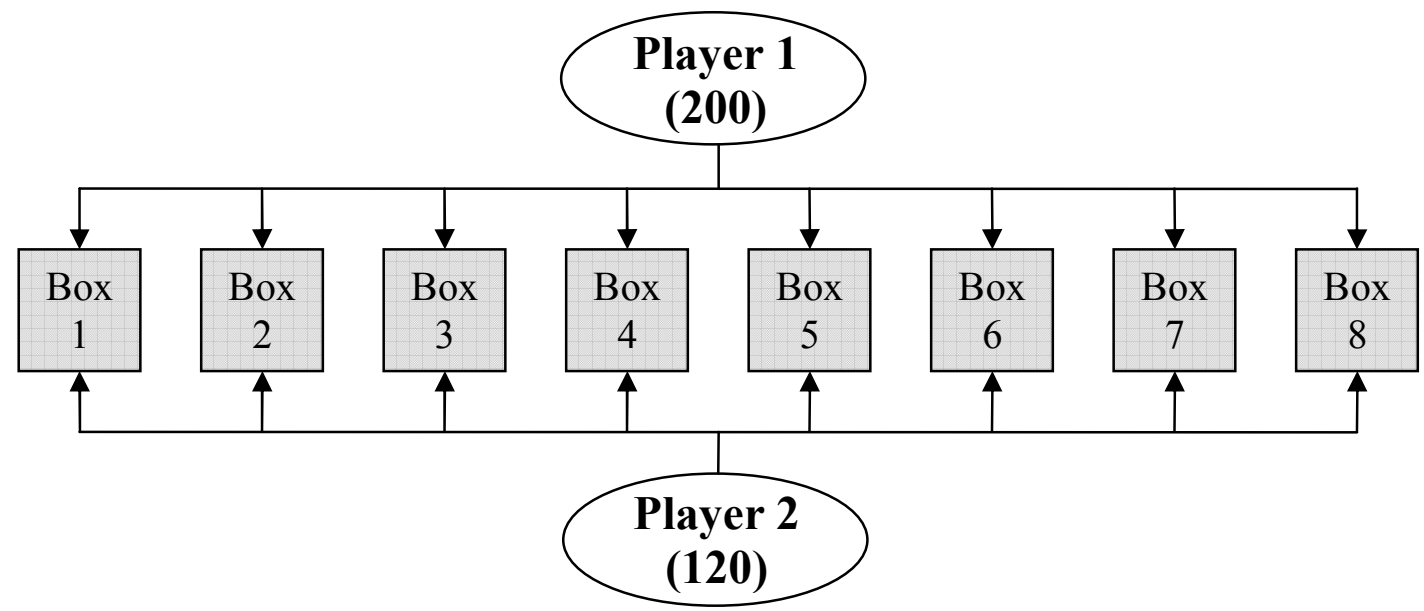

Figure 2: Distribution of Tokens in the Lottery Treatment
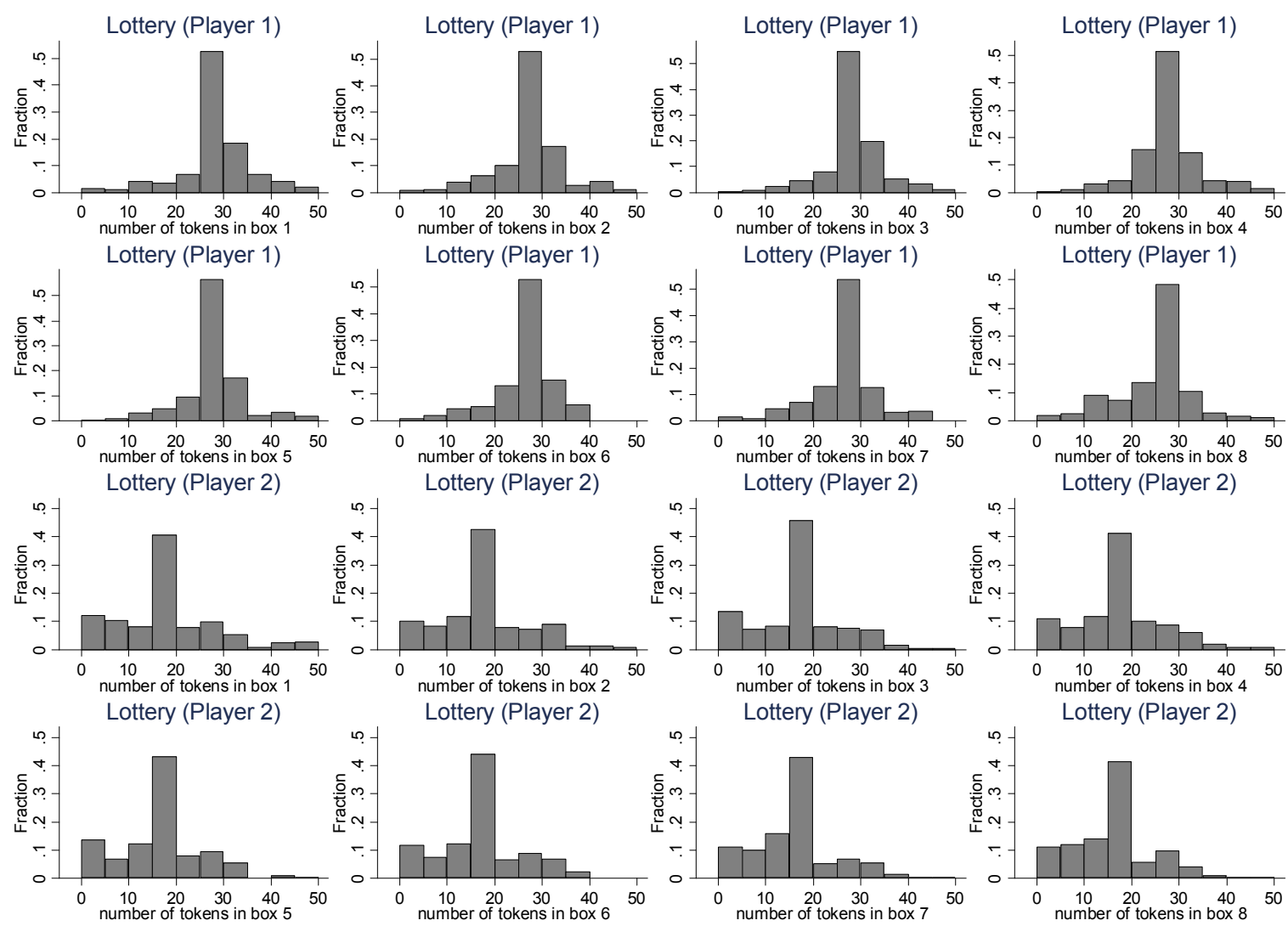
Figure 3: Distribution of Tokens in the Auction Treatment
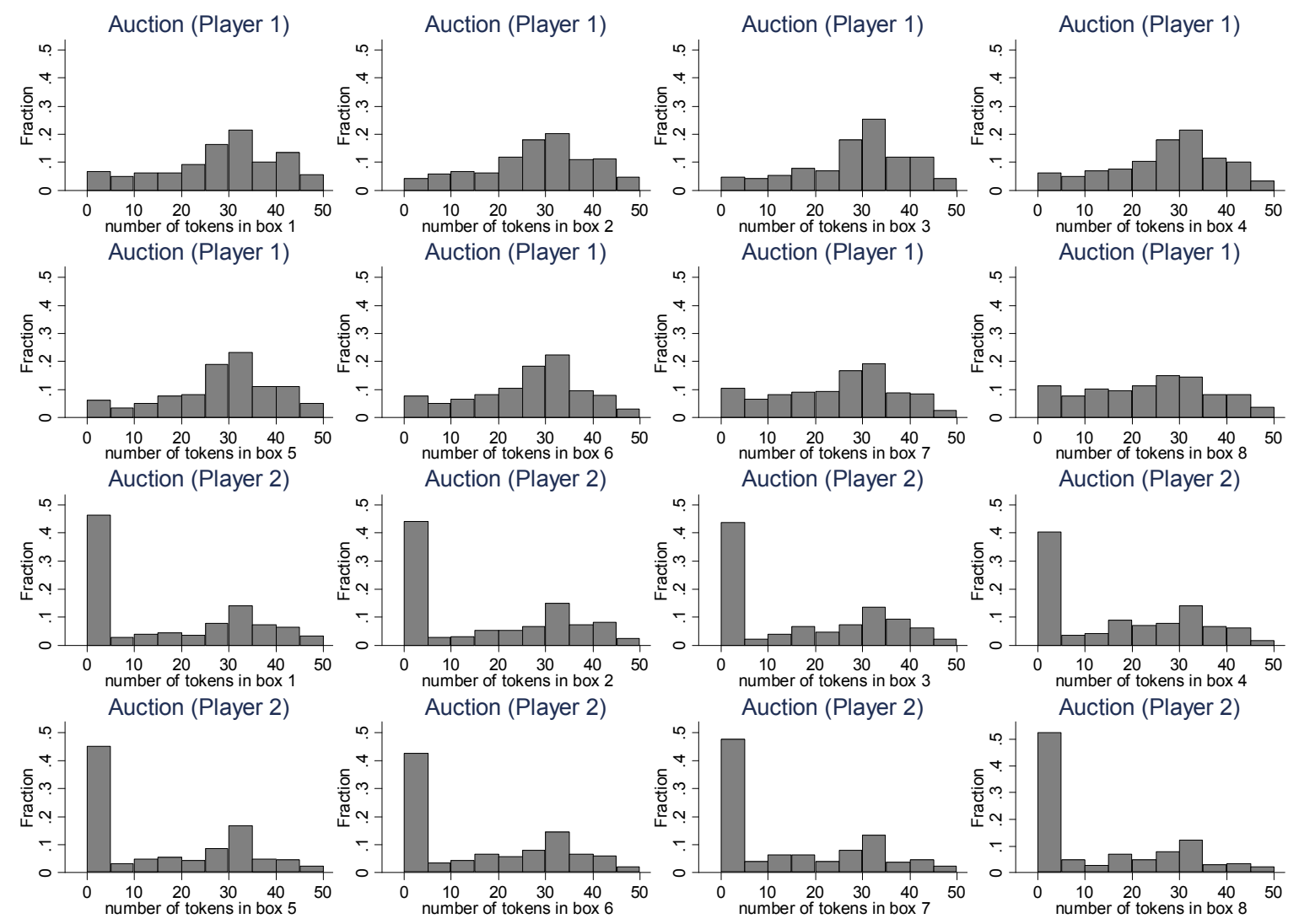

Figure 3: Fraction of Players who use Decimals
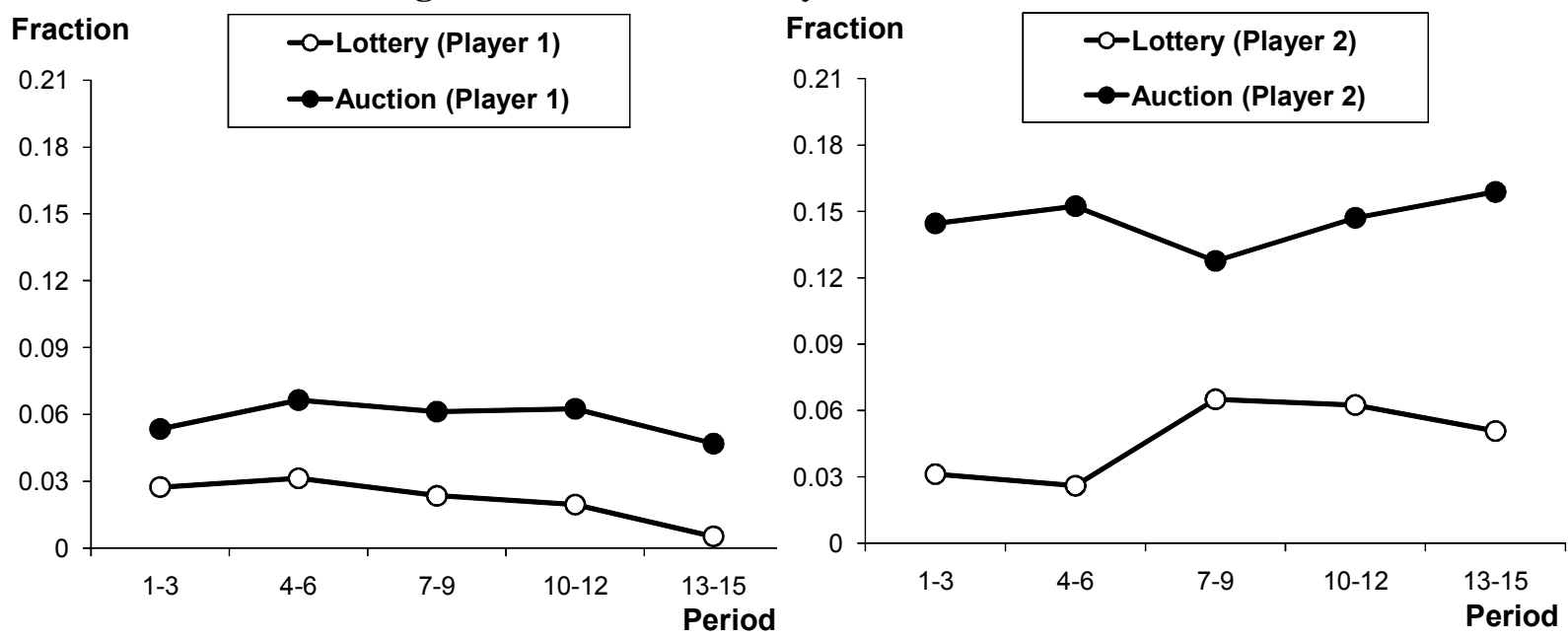
Figure 4: Allocation by Player 1 in the Auction Treatment (Strangers)

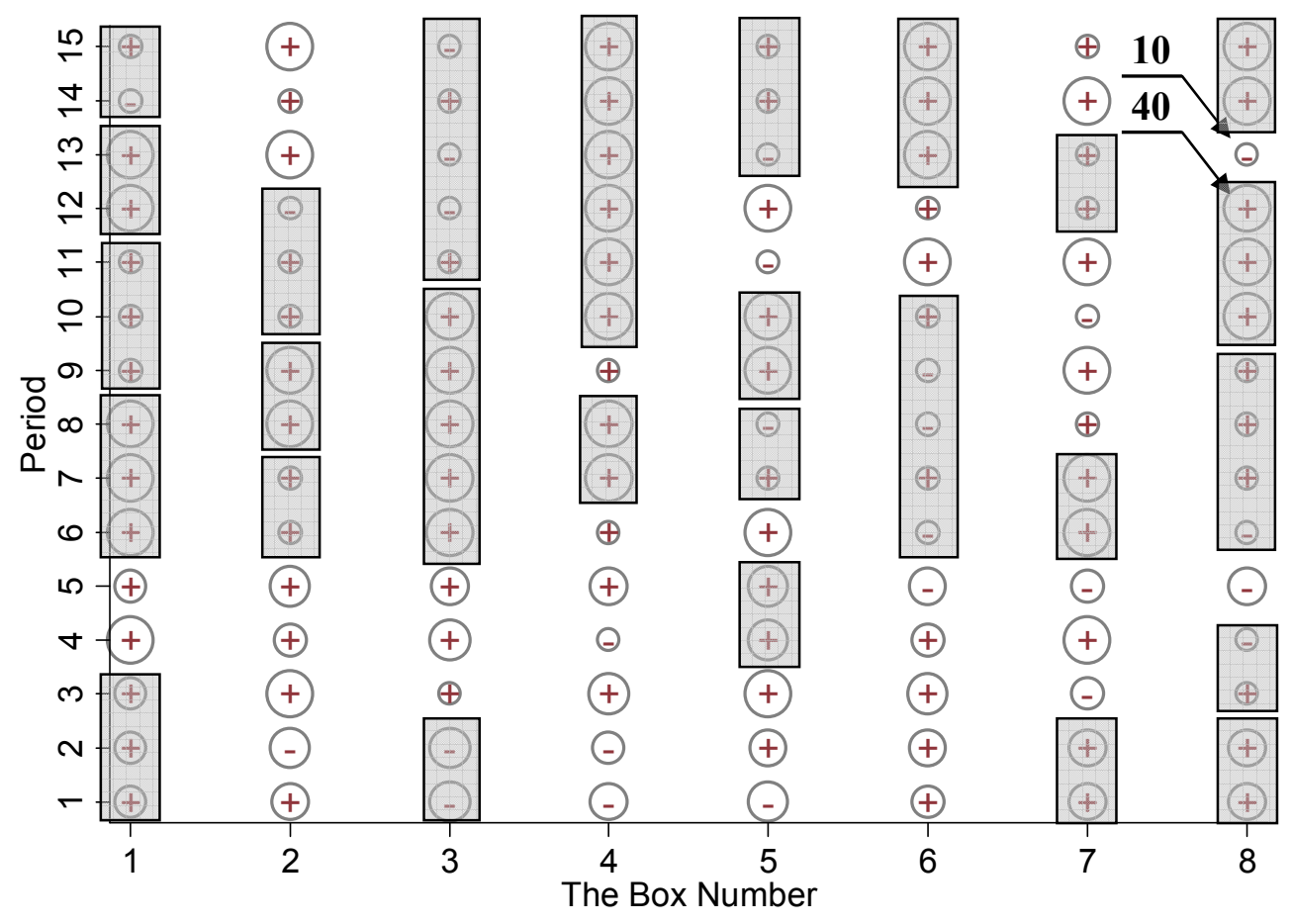

Figure 5: Allocation by Player 1 in the Auction Treatment (Partners)

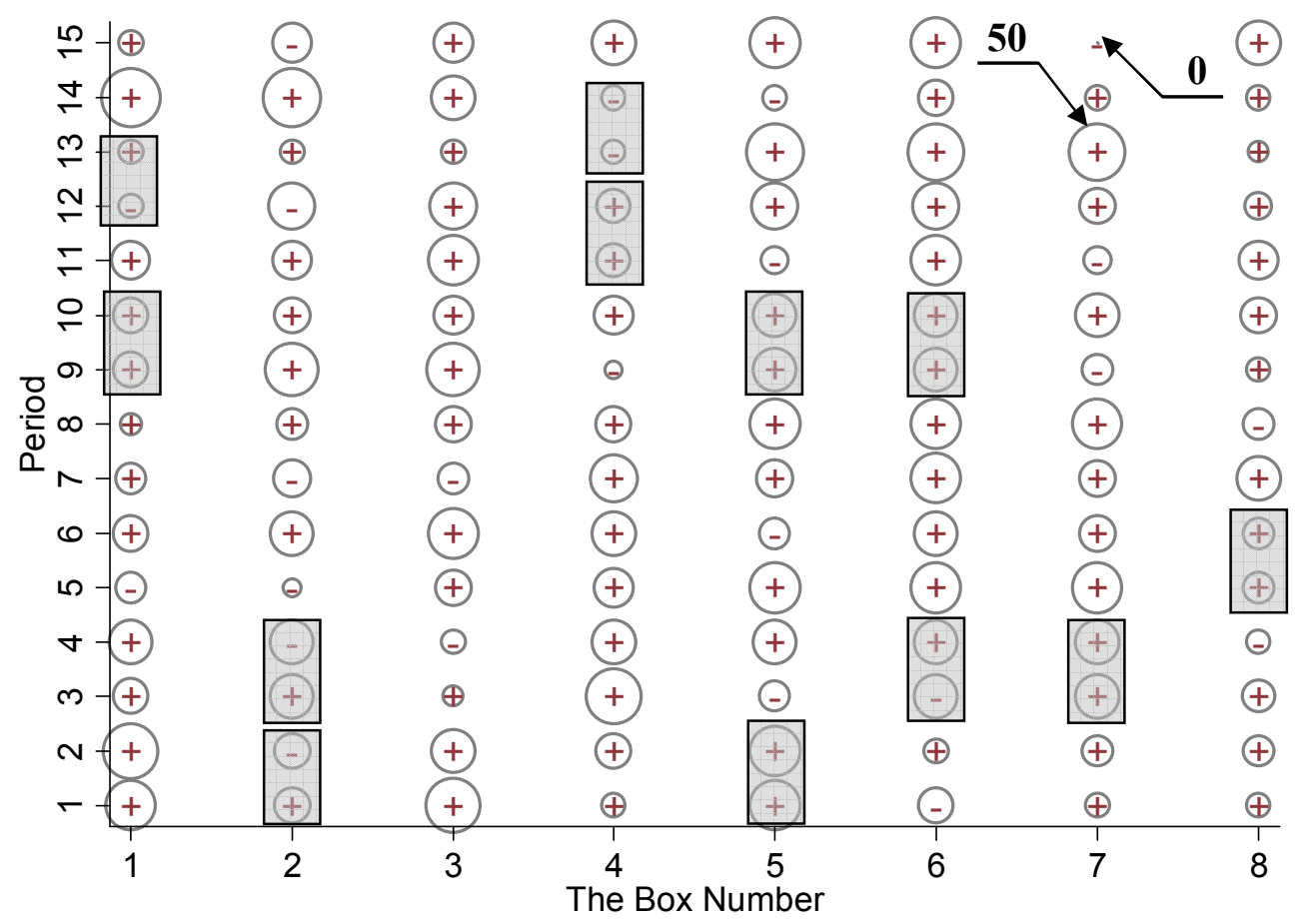


Figure 6: Cumulative Distribution of Differences in the Auction Treatment

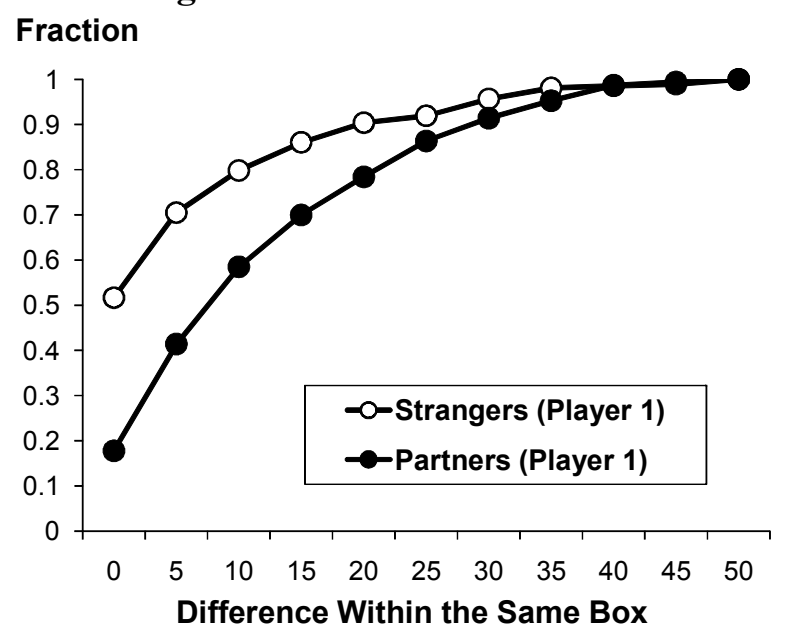
Fraction

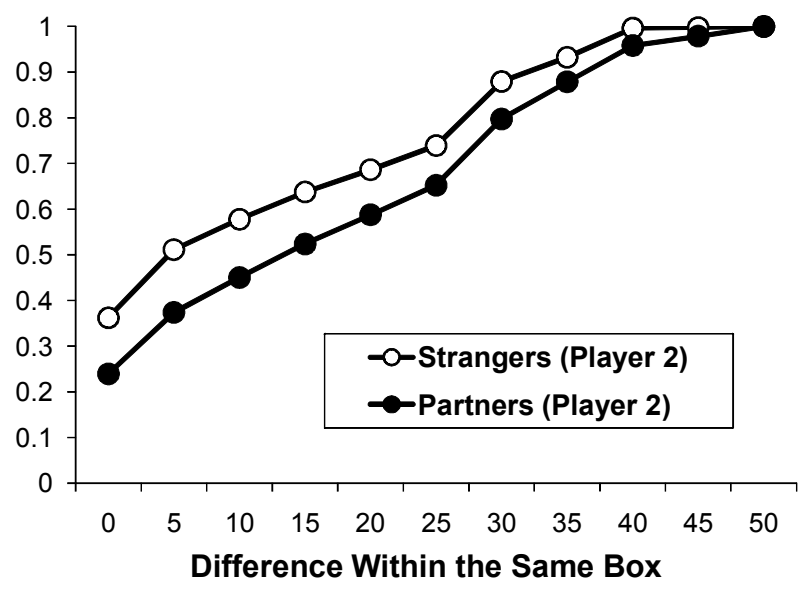




\section{Appendix}

\section{GENERAL INSTRUCTIONS}

This is an experiment in the economics of strategic decision making. Various research agencies have provided funds for this research. The instructions are simple. If you follow them closely and make careful decisions, you can earn an appreciable amount of money.

The experiment will proceed in three parts. Each part contains decision problems that require you to make a series of economic choices which determine your total earnings. The currency used in Part 1 of the experiment is U.S. Dollars. The currency used in Parts 2 and 3 of the experiment is francs. Francs will be converted to U.S. Dollars at a rate of $\underline{\mathbf{X}}$ francs to $\underline{\mathbf{1}}$ dollar. At the end of today's experiment, you will be paid in private and in cash. 16 participants are in today's experiment.

It is very important that you remain silent and do not look at other people's work. If you have any questions, or need assistance of any kind, please raise your hand and an experimenter will come to you. If you talk, laugh, exclaim out loud, etc., you will be asked to leave and you will not be paid. We expect and appreciate your cooperation.

At this time we proceed to Part 1 of the experiment.

\section{INSTRUCTIONS FOR PART 1}

\section{YOUR DECISION}

In this part of the experiment you will be asked to make a series of choices in decision problems. How much you receive will depend partly on chance and partly on the choices you make. The decision problems are not designed to test you. What we want to know is what choices you would make in them. The only right answer is what you really would choose.

For each line in the table in the next page, please state whether you prefer option A or option B. Notice that there are a total of $\mathbf{1 5}$ lines in the table but just one line will be randomly selected for payment. Each line is equally likely to be chosen, so you should pay equal attention to the choice you make in every line. After you have completed all your choices a token will be randomly drawn out of a bingo cage containing tokens numbered from 1 to 15. The token number determines which line is going to be paid.

Your earnings for the selected line depend on which option you chose: If you chose option A in that line, you will receive $\mathbf{\$ 1}$. If you chose option B in that line, you will receive either $\mathbf{\$ 3}$ or $\mathbf{\$ 0}$. To determine your earnings in the case you chose option B there will be second random draw. A token will be randomly drawn out of the bingo cage now containing twenty tokens numbered from 1 to 20. The token number is then compared with the numbers in the line selected (see the table). If the token number shows up in the left column you earn $\$ 3$. If the token number shows up in the right column you earn $\$ 0$.

Are there any questions?

\begin{tabular}{|c|c|c|c|c|}
\hline $\begin{array}{l}\text { Deci } \\
\text { sion } \\
\text { no. }\end{array}$ & $\begin{array}{l}\text { Opti } \\
\text { on A }\end{array}$ & & $\begin{array}{c}\text { Option } \\
\text { B }\end{array}$ & $\begin{array}{l}\text { Please } \\
\text { choose } \\
\text { A or B }\end{array}$ \\
\hline 1 & $\$ 1$ & \$3 never & \$0 if $1,2,3,4,5,6,7,8,9,10,11,12,13,14,15,16,17,18,19,20$ & \\
\hline 2 & $\$ 1$ & $\mathbf{\$ 3}$ if 1 comes out of the bingo cage & \$0 if $2,3,4,5,6,7,8,9,10,11,12,13,14,15,16,17,18,19,20$ & \\
\hline 3 & $\$ 1$ & $\$ 3$ if 1 or 2 & \$0 if $3,4,5,6,7,8,9,10,11,12,13,14,15,16,17,18,19,20$ & \\
\hline 4 & $\$ 1$ & $\mathbf{\$ 3}$ if $1,2,3$ & $\mathbf{\$ 0}$ if $4,5,6,7,8,9,10,11,12,13,14,15,16,17,18,19,20$ & \\
\hline 5 & $\$ 1$ & $\mathbf{\$ 3}$ if $1,2,3,4$ & $\$ 0$ if $5,6,7,8,9,10,11,12,13,14,15,16,17,18,19,20$ & \\
\hline 6 & $\$ 1$ & $\mathbf{\$ 3}$ if $1,2,3,4,5$ & $\$ 0$ if $6,7,8,9,10,11,12,13,14,15,16,17,18,19,20$ & \\
\hline 7 & $\$ 1$ & $\mathbf{\$ 3}$ if $1,2,3,4,5,6$ & \$0 if $7,8,9,10,11,12,13,14,15,16,17,18,19,20$ & \\
\hline 8 & $\$ 1$ & $\mathbf{\$ 3}$ if $1,2,3,4,5,6,7$ & \$0 if $8,9,10,11,12,13,14,15,16,17,18,19,20$ & \\
\hline 9 & $\$ 1$ & $\mathbf{\$ 3}$ if $1,2,3,4,5,6,7,8$ & \$0 if $9,10,11,12,13,14,15,16,17,18,19,20$ & \\
\hline 10 & $\$ 1$ & $\mathbf{\$ 3}$ if $1,2,3,4,5,6,7,8,9$ & \$0 if $10,11,12,13,14,15,16,17,18,19,20$ & \\
\hline 11 & $\$ 1$ & $\mathbf{\$ 3}$ if $1,2,3,4,5,6,7,8,9,10$ & $\mathbf{\$ 0}$ if $11,12,13,14,15,16,17,18,19,20$ & \\
\hline 12 & $\$ 1$ & $\mathbf{\$ 3}$ if $1,2,3,4,5,6,7,8,9,10,11$ & $\mathbf{\$ 0}$ if $12,13,14,15,16,17,18,19,20$ & \\
\hline 13 & $\$ 1$ & $\mathbf{\$ 3}$ if $1,2,3,4,5,6,7,8,9,10,11,12$ & $\$ 0$ if $13,14,15,16,17,18,19,20$ & \\
\hline 14 & $\$ 1$ & $\$ \mathbf{3}$ if $1,2,3,4,5,6,7,8,9,10,11,12,13$ & \$0 if $14,15,16,17,18,19,20$ & \\
\hline 15 & $\$ 1$ & $\mathbf{\$ 3}$ if $1,2,3,4,5,6,7,8,9,10,11,12,13,14$ & \$0 if $15,16,17,18,19,20$ & \\
\hline
\end{tabular}




\section{INSTRUCTIONS FOR PART 2 \\ YOUR DECISION}

The second part of the experiment consists of $\mathbf{1 5}$ decision-making periods. At the beginning of each period, you will be randomly and anonymously placed into a group which consists of two participants: participant 1 and participant 2. At the beginning of the first period you will be randomly assigned either as participant 1 or as participant 2. You will remain in the same role assignment throughout the entire experiment. So, if you are assigned as participant 1 then you will stay as participant 1 throughout the entire experiment.

Each period, participant 1 will receive 200 tokens and participant 2 will receive 120 tokens. Both participants will choose how to allocate their tokens to 8 boxes, as shown on a decision screen below.

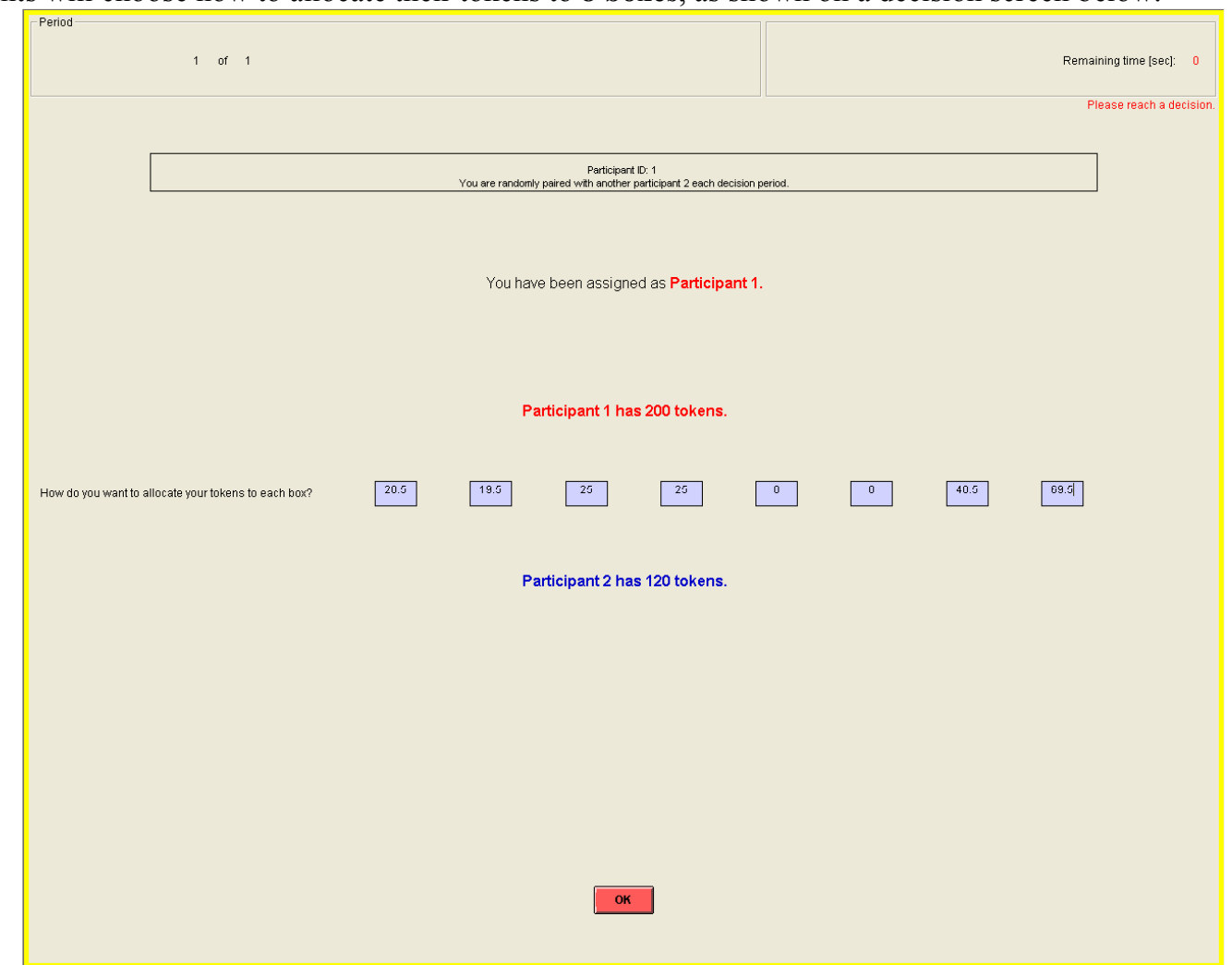

Participant 1 can allocate any number of tokens between $\mathbf{0}$ and $\mathbf{2 0 0}$ (including 0.5 decimal points) to each box. The total number of tokens in all boxes must sum to $\mathbf{2 0 0}$ or the computer will not accept the decision of participant 1. Similarly, participant 2 can allocate any number of tokens between $\mathbf{0}$ and $\mathbf{1 2 0}$ (including 0.5 decimal points). The total number of tokens in all boxes must sum to $\mathbf{1 2 0}$ or the computer will not accept the decision of participant 2 .

\section{YOUR EARNINGS}

After each participant has made his or her decisions, your earnings for the period are calculated. Your period earnings are proportional to the number of boxes you win. For each box you win you will receive 1 franc.

\section{Your earnings $=$ Number of boxes you won $\times 1$ franc}

So, if you win all 8 boxes, you will receive 8 francs for this period. If you do not win any of the boxes, you will receive 0 francs. Francs will be converted to U.S. Dollars at a rate of $\underline{\mathbf{X}}$ francs to $\underline{\mathbf{1}}$ dollar. Your conversion rates are your private information. All conversion rates for participant 1 are equal and all conversion rates for participant 2 are equal. However, the conversion rates are different for participants 1 and 2. Notice that the more francs you earn, the more dollars you earn. What you earn depends partly on your decision and partly on the decision of the other participant with whom you are paired.

The more tokens you allocate to a particular box, the more likely you are to win that box. The more tokens the other participant allocates to the same box, the less likely you are to win that box. Specifically, for each token you allocate to a particular box you will receive $\mathbf{1 0}$ lottery tickets. At the end of each period the computer draws 
randomly one ticket among all the tickets purchased by you and the other participant in your group. The owner of the drawn ticket wins the box and receives 1 franc for that box. Thus, your chance of winning a particular box is given by the number of tokens you allocate to that box divided by the total number of tokens you and the other participant allocate to that box.

Chance of $\quad=\quad$ Number of tokens you allocate to that box winning a box $=$ Number of tokens you allocate + Number of tokens the other participant allocates to that box

In case both participants allocate zero to the same box, the computer will randomly chose a winner of that box. Therefore, each participant has the same chance of winning the box.

\section{Example of the Random Draw}

This is a hypothetical example used to illustrate how the computer makes a random draw. Let's say participant 1 and participant 2 allocate their tokens to eight boxes in the following way.

\begin{tabular}{|c|c|r|r|r|}
\hline Box & Participant 1 & Participant 2 & $\begin{array}{r}\text { Chance of winning the box } \\
\text { for Participant 1 }\end{array}$ & $\begin{array}{r}\text { Chance of winning the box } \\
\text { for Participant } 2\end{array}$ \\
\hline 1 & 20.5 & 15 & $20.5 /(20.5+15)=0.58$ & $15 /(20.5+15)=0.42$ \\
2 & 19.5 & 15 & $19.5 /(19.5+15)=0.57$ & $15 /(19.5+15)=0.43$ \\
3 & 25 & 10 & $25 /(25+10)=0.71$ & $25 /(25+10)=0.29$ \\
4 & 25 & 10 & $25 /(25+10)=0.71$ & $25 /(25+10)=0.29$ \\
5 & 0 & 0 & 0.50 & 0.50 \\
6 & 0 & 40 & $40.5 /(40.5+15.5)=0.72$ & $15.5 /(40.5+15.5)=0.28$ \\
7 & 40.5 & 15.5 & $69.5 /(69.5+14.5)=0.83$ & $14.5 /(69.5+14.5)=0.17$ \\
\hline 8 & 69.5 & 14.5 & & \\
\hline Total & 200 & 120 & & \\
\hline
\end{tabular}

Participant 1 allocates 20.5 tokens to box $1,19.5$ tokens to box 2, 25 tokens box 3, 25 tokens to box 4, 0 tokens to box 5, 0 tokens to box 6, 40.5 tokens to box 7, and 69.5 tokens to box 8 (total of 200 tokens). Participant 2 allocates 15 tokens to box 1,15 tokens to box 2,10 tokens to box 3,10 tokens to box 4,0 tokens to box 5,40 tokens to box $6,15.5$ tokens to box 7, and 14.5 tokens to box 8 (total of 120 tokens). Therefore, the computer will assigns lottery tickets to participant 1 and to participant 2 according to their allocation of tokens.

For example, in box 1, the computer will assign 205 lottery tickets to participant 1 and 150 lottery tickets to participant 2. Then the computer will randomly draw one lottery ticket out of $355(205+150)$. As you can see, participant 1 has a higher chance of winning box 1: 20.5/(20.5+15) $=0.58$. Participant 2 has lower chance of winning box 1: $15 /(20.5+15)=0.42$.

Similarly, in box 6 , the computer will assign 0 lottery tickets to participant 1 and 400 lottery tickets to participant 2. Then the computer will randomly draw one lottery ticket out of $400(0+400)$. As you can see, participant 1 has no chance of winning box $6: 0 /(0+40)=0.0$. Therefore, participant 2 will win box 6 for sure: $40 /(0+40)=1.0$.

After all participants allocate their tokens and press the OK button, the computer will make a random draw for each box separately and independently. Note that you can never guarantee that you will win a particular box. However, by increasing your allocation to that box, you can increase your chance of winning that box. The random draw made by the computer will decide which boxes you win. Then the computer will calculate your period earnings based on how many boxes you won.

At the end of each period, the allocation of your tokens, the allocation of the other participant's tokens, which boxes you win, your period earnings, and your cumulative earnings are reported on the outcome screen as shown below. Once the outcome screen is displayed you should record your results for the period on your Personal Record Sheet under the appropriate heading. 


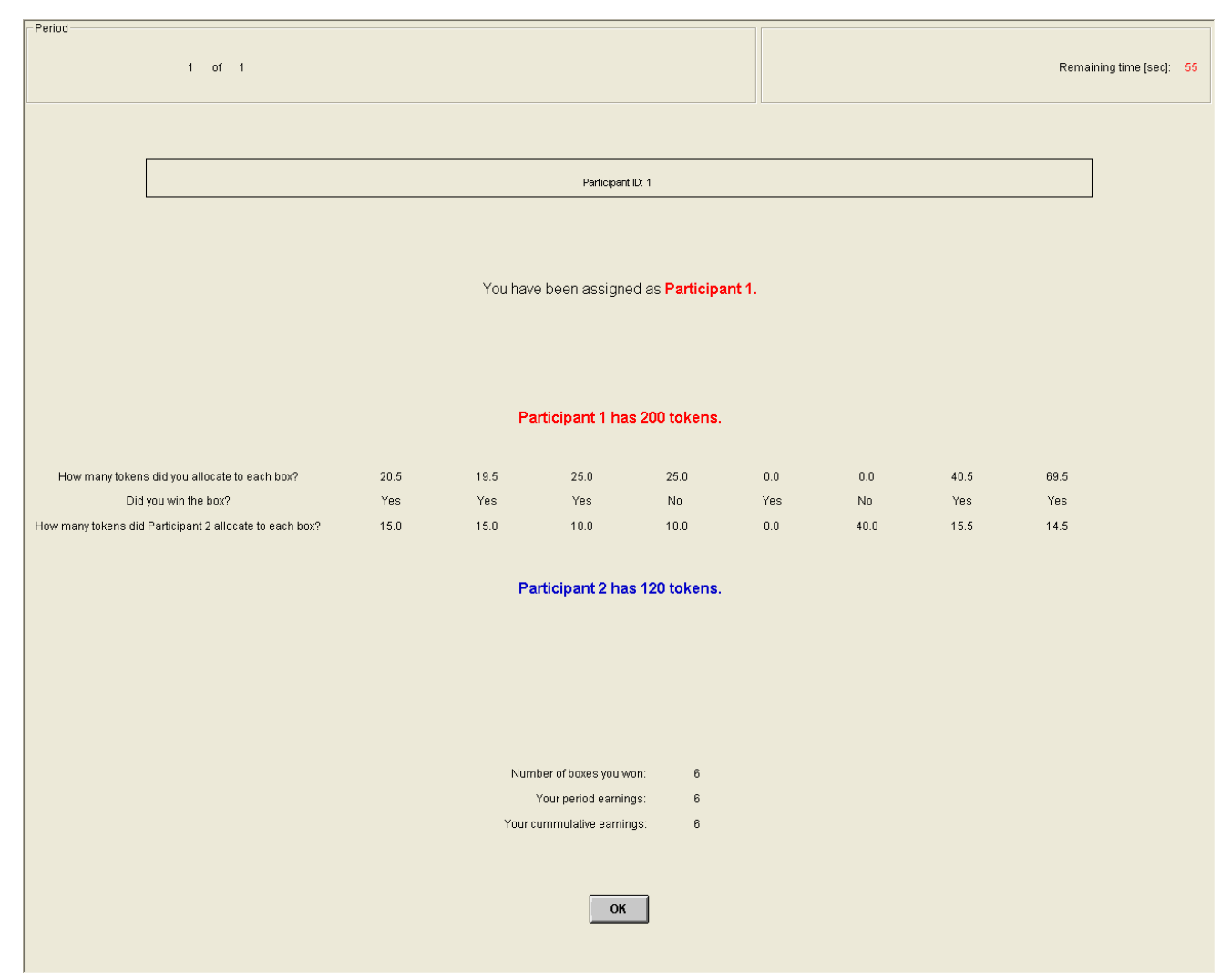

\section{IMPORTANT NOTES}

At the beginning of the first period you will be randomly assigned either as participant 1 or as participant 2 . You will remain in the same role assignment throughout the entire experiment. Each consecutive period you will be randomly re-paired with another participant of opposite assignment. So, if you are participant 1, each period you will be randomly re-paired with another participant 2 . If you are participant 2 , each period you will be randomly repaired with another participant 1.

At the end of the experiment you will convert your cumulative earnings into a payment in U.S. dollars. Your conversion rates are your private information. All conversion rates for participant 1 are equal and all conversion rates for participant 2 are equal. However, the conversion rates are different for participants 1 and 2.

\section{Are there any questions?}

\section{INSTRUCTIONS FOR PART 3}

The third part of the experiment consists of $\mathbf{1 5}$ decision-making periods. As in the previous part 2 of the experiment, you will be placed into a group which consists of two participants: participant 1 and participant 2. Your assignment as participant 1 or participant 2 will be the same as it was in the previous part 2 of the experiment.

Each period, participant 1 will receive $\mathbf{2 0 0}$ tokens and participant 2 will receive $\mathbf{1 2 0}$ tokens. Both participants will choose how to allocate their tokens to 8 boxes. Participant 1 can allocate any number of tokens between $\mathbf{0}$ and 200 (including 0.5 decimal points) to each box. The total number of tokens in all boxes must sum to $\mathbf{2 0 0}$ or the computer will not accept the decision of participant 1 . Similarly, participant 2 can allocate any number of tokens between $\mathbf{0}$ and 120 (including 0.5 decimal points). The total number of tokens in all boxes must sum to 120 or the computer will not accept the decision of participant 2.

After each participant has made his or her decisions, your earnings for the period are calculated. Your period earnings are proportional to the number of boxes you win. For each box you win you will receive 1 franc.

Your earnings $=$ Number of boxes you won $\times 1$ franc

The main difference from part 2 is that the computer will choose the winner of each box in the following way. The participant who allocates more tokens than the other participant to a particular box wins that box with certainty. So, if participant 1 allocates 30 tokens to a particular box while participant 2 allocates 29.5 tokens to the 
same box then the computer will chose participant 1 as the winner of that box. In case both participants allocate the same amount to the same box, the computer will always chose participant 1 as a winner of that box. In case both participants allocate zero to the same box, the computer will always chose participant 1 as a winner of that box.

After all participants allocate their tokens and press the OK button, the computer will determine the winner of each box and will calculate your period earnings based on how many boxes you won.

At the end of each period, the allocation of your tokens, the allocation of the other participant's tokens, which boxes you win, your period earnings, and your cumulative earnings are reported on the outcome screen. Once the outcome screen is displayed you should record your results for the period on your Personal Record Sheet under the appropriate heading.

\section{IMPORTANT NOTES}

Your assignment as participant 1 or participant 2 will be the same as it was in the previous part 2 of the experiment. You will remain in the same role assignment throughout the entire experiment. Each consecutive period you will be randomly re-paired with another participant of opposite assignment. So, if you are participant 1, each period you will be randomly re-paired with another participant 2. If you are participant 2, each period you will be randomly re-paired with another participant 1.

The participant who allocates more tokens than the other participant to a particular box wins that box with certainty.

At the end of the experiment you will convert your cumulative earnings into a payment in U.S. dollars. Your conversion rates are your private information. All conversion rates for participant 1 are equal and all conversion rates for participant 2 are equal. However, the conversion rates are different for participants 1 and 2 .

Are there any questions? 